\title{
Fluctuation-induced tunneling conduction in disordered materials
}

\author{
Ping Sheng* \\ RCA Laboratories, Princeton, New Jersey 08540
}

(Received 30 April 1979)

\begin{abstract}
In disordered materials generally characterized by large conducting regions (or long conducting pathways) separated by small insulating barriers, it is shown that the electrical conduction can be ascribed to a novel mechanism, fluctuation-induced tunneling, in which the thermally activated voltage fluctuations across insulating gaps play an important role in determining the temperature and field dependences of the conductivity. By considering the modulating effects induced by voltage fluctuations on either an image-force corrected rectangular potential barrier or a parabolic barrier, a theoretical expression for the tunneling conductivity is derived which displays thermally activated characteristics at high temperatures but becomes identical to the temperature-independent simple elastic tunneling at low temperatures. Between the two limiting behaviors the temperature dependence of the conductivity is controlled by the shape of the tunneling barrier. An expression for the high-field tunneling current is similarly obtained. It is found that, while the tunneling current increases as a nonlinear function of the field, the degree of nonlinearity decreases as the temperature increases, indicating an effective lowering and narrowing of the barrier by voltage fluctuations. The theory is also generalized from the consideration of a single tunnel junction to a random network of tunnel junctions by the application of the effective-medium theory. The theoretical predictions are compared with the experimental results for three disordered systems: (1) carbonpolyvinylchloride composites, (2) heavily doped, closely compensated GaAs, and (3) doped polyacetylene $(\mathrm{CH})_{x}$ in the metallic regime. In each case excellent agreement is obtained. It is shown in particular that the nonmetallic temperature dependence of the resistivity in doped metallic $(\mathrm{CH})_{x}$ samples can be understood in terms of the present theory.
\end{abstract}

\section{INTRODUCTION}

Hopping transport of electrons between localized sites has received considerable attention in recent years as the mechanism responsible for the characteristic $\exp \left(-\mathrm{const} / T^{\alpha}\right)$ form of temperature dependence of conductivity observed in amorphous semiconductors ${ }^{1}\left(\alpha=\frac{1}{4}\right)$ and sputtered granular metal films ${ }^{2}\left(\alpha=\frac{1}{2}\right)$. However, there are a variety of disordered materials, such as some conductorinsulator composites, ${ }^{3}$ disordered semiconductors, ${ }^{4}$ and doped organic semiconductors, ${ }^{5,6}$ in which most of the conduction electrons are delocalized and free to move over distances very large as compared to the atomic dimension. For these random systems the electrical conduction is dominated by electron transfer between large conducting segments rather than by hopping between localized sites. It is the purpose of this paper to present a new conduction mechanism, fluctuation-induced tunneling, which characterizes such electron transfers across the insulating gaps in the conducting pathways. The essential physics of the new mechanism is contained in the observation that, since the electrons tend to tunnel between conducting regions at points of their closest approach, the relevant tunnel junctions are usually small in size and are therefore subject to large (thermally activated) voltage fluctuations across the junction. By modulating the potential barrier, the voltage fluctuations directly influence the tunneling probability and introduce a characteristic temperature variation to the normally temperature-independent tunneling conductivity.

The voltage fluctuations and their probability of occurrence are considered in Sec. II. The calculation of fluctuation-induced tunneling characteristics for a single tunnel junction, and the generalization of the theory to a random network of tunnel junctions, are presented in Sec. III and IV, respectively. In Sec. $\mathrm{V}$ the theory developed is applied to three disordered systems: (1) carbonpolyvinylchloride (C-PVC) composites, (2) heavily doped, closely compensated GaAs, and (3) doped polyacetylene $(\mathrm{CH})_{x}$. In all three cases the predictions of the theory are found to be in excellent accord with the experimental results.

\section{VOLTAGE FLUCTUATIONS}

Consider a region of close approach between two large conducting segments in a disordered material as shown in Fig. 1(a). Since the electron tunneling probability depends exponentially on the insulating barrier thickness, it is expected that practically all the tunneling occurs within the small surface areas delineated schematically by heavy lines in Fig. 1(a). We can approximate such a tunnel junction by a parallel-plate capacitor with area $A$, separation $w$, and capacitance $C=A / 4 \pi w$, as shown in Fig. 1(b), where $R / 2$ denotes the resistance connecting the junction capacitor to the 

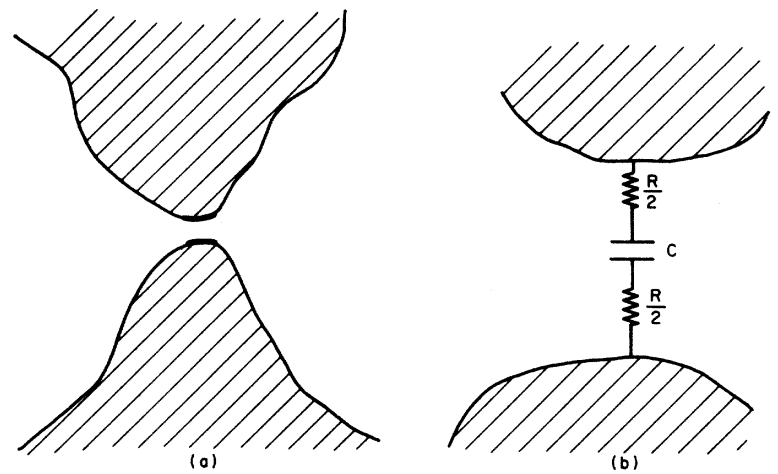

FIG. 1. A region of close approach between two conducting segments. Shaded area denotes conductors. In (a), the heavy lines delineate the surface areas within which most of the tunneling occurs. This tunnel junction is schematically depicted as a parallel-plate capacitor in (b). $R$ and $C$ are defined in the text.

rest of the conducting segment. Of course, $C$ is only a small part of the total capacitance $C_{0}$ between the two large conducting segments. A direct consequence of the large size of the conducting regions is that $C_{0}$ is large and therefore the charging energy, $e^{2} / 2 C_{0}$, required to transfer an electron of charge $e$ from one neutral conducting region to its neighbor, is completely negligible. This is in sharp distinction to conduction in granular metals, ${ }^{2}$ where charging energy plays the dominant role in determining the electrical transport properties.

Owing to the random thermal motion of electrons in the conducting region, there can be transient excess or deficit of charges on the tunnel junction surfaces, resulting in voltage fluctuations across the junction. In order to calculate the magnitude of these fluctuations, let us represent the two conducting segments and the tunnel junction shown in Fig. 1(b) by the equivalent circuit of Fig. 2, where the noise source is identified as the Johnson noise voltage generator with ${ }^{7}\left\langle V_{f}^{2}\right\rangle=4 k T R d f$, where $V_{f}$ is the fluctuation voltage of a resistor in a

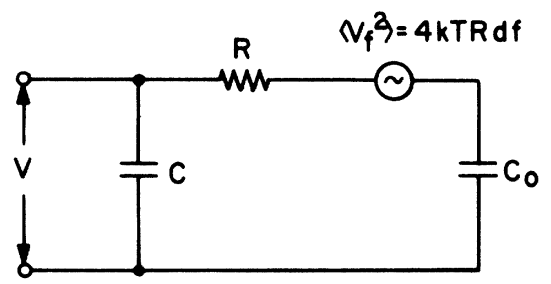

FIG. 2. The equivalent circuit from which the fluctuation voltage across $C$ is calculated [see Eq. (1a)]. Since $C$ is a part of the total capacitance between the two conducting segments, $C$ and $C_{0}$ are in parallel to each other. small frequency interval $d f,\langle\rangle$ denotes thermal averaging, and $k$ is Boltzmann's constant. The averaged square of the thermal fluctuation voltage across the tunnel junction, $\left\langle V_{T}^{2}\right\rangle$, can now be simply calculated $^{8}$ as

$$
\left\langle V_{T}^{2}\right\rangle=\int_{0}^{\infty} \frac{4 k T R d f}{(2 \pi f C R)^{2}+\left(1+C / C_{0}\right)^{2}} .
$$

Since $C \ll C_{0}$, the $C / C_{0}$ term in the integrand can be neglected, resulting in

$$
\left\langle V_{T}^{2}\right\rangle=k T / C \text {. }
$$

Equation (1b) states the familiar result that $\left\langle V_{T}^{2}\right\rangle$ is inversely proportional to the capacitance of the junction. Since the internal tunnel junctions in disordered materials are usually small in area as noted before, the resulting large voltage fluctuations across the junctions are expected to play an important role in modifying the electron tunneling probability.

Equation (1b) can be alternatively derived by considering the capacitor as a system with one degree of freedom and applying the equipartition theorem ${ }^{9}$

$$
\frac{1}{2} C\left\langle V_{T}^{2}\right\rangle=\frac{1}{2} k T .
$$

Since the equipartition theorem is a direct consequence of the Boltzmann distribution, Eq. (2) suggests that the probability of fluctuations is proportional to $\exp (-\Delta E / k T), \Delta E$ being the energy necessary to move the system away from equilibrium. For the general case of a capacitor with an externally applied potential $V_{A}$, a deviation of $\pm V_{T}$ away from $V_{A}$ requires

$$
\Delta E=\frac{1}{2} C\left(V_{A} \pm V_{T}\right)^{2}-\frac{1}{2} C V_{A}^{2} \mp C V_{T} V_{A},
$$

where the first two terms represent the change in the electrostatic energy of the capacitor and the last term gives the work done by the external potential. The net result of $\Delta E=\frac{1}{2} C V_{T}^{2}$ means that the fluctuation probability function ${ }^{10}$ is given by

$$
P\left(V_{T}\right)=(2 C / \pi k T)^{1 / 2} \exp \left(-\frac{1}{2} C V_{T}^{2} / k T\right) .
$$

Here the pre-exponential factor normalizes the integral of $P\left(V_{T}\right)$ for $0 \leqslant V_{T}<\infty$. It is sometimes convenient for subsequent calculation to express the fluctuation probability function in terms of a fluctuating electric field. By changing the variable from $V_{T}$ to $\mathcal{E}_{T} \equiv V_{T} / w$, Eq. (3b) is easily rewritten as

$$
P\left(\mathcal{E}_{T}\right)=\left(\frac{4 a}{\pi k T}\right)^{1 / 2} \exp \left(-a \mathcal{E}_{T}^{2} / k T\right), \quad 0 \leqslant \mathcal{E}_{T}<\infty,
$$

where $a \equiv w A / 8 \pi$ is a measure of the junction volume. From $P\left(V_{T}\right)$ [or $P\left(\mathcal{E}_{T}\right)$ ] one can calculate the 
thermal average for any function of $V_{T}$ (or $\mathcal{E}_{T}$ ). In particular, we note that the value of $\left\langle V_{T}^{2}\right\rangle$, evaluated by

$$
\left\langle V_{T}^{2}\right\rangle=\int_{0}^{\infty} V_{T}^{2} P\left(V_{T}\right) d V_{T}=k T / C,
$$

is consistent with the result obtained earlier.

\section{SINGLE-JUNCTION CHARACTERISTICS}

From the discussion of Sec. II we have seen that at any tunnel junction between conducting pathways there are two possible sources of the electric field: the thermal fluctuating field $\mathcal{E}_{T}$ and the applied field $\mathcal{E}_{A}$. The field $\mathcal{E}_{A}$, however, has to be distinguished from the macroscopic average of the applied field. Owing to the exclusion of electric field from conducting regions and the resulting concentration of voltage drops at the junctions, it is expected that $\mathcal{E}_{A}$ would be (1) a factor $M$ larger than the macroscopic average field, where $M$ is roughly the ratio between the average size of the conducting segments and the average junction width, and (2) parallel to the tunneling direction (and the thermal fluctuating field $\mathcal{E}_{T}$ ) regardless of the junction orientation relative to the macroscopic average field direction. Since it is equally probable for the thermal fluctuating field to be in the same direction of $\mathcal{E}_{A}$ as opposite to it, a junction can experience two values of the total field, $\mathcal{E}_{A}+\mathcal{E}_{T}$ and $\mathcal{E}_{A}-\mathcal{E}_{T}$, for any given value of $\left|\mathcal{E}_{T}\right|$. If $\mathcal{E}_{A}<\left|\mathcal{E}_{T}\right|$, the two resulting tunneling current densities, $\frac{1}{2} j\left(\mathcal{E}_{A}+\mathcal{E}_{T}\right)$ and $\frac{1}{2} j\left(\mathcal{E}_{A}-\mathcal{E}_{T}\right)$, are in opposite directions (the factor $\frac{1}{2}$ is present because each has equal probability of occurrence), and the net tunneling current along the direction of the applied field is given by

$$
\Delta j=\frac{1}{2}\left[j\left(\mathcal{E}_{T}+\mathcal{E}_{A}\right)-j\left(\mathcal{E}_{T}-\mathcal{E}_{A}\right)\right] .
$$

From Eq. (4) a partial conductivity $\Sigma\left(\mathcal{E}_{T}\right)$ can be defined as

$$
\Sigma\left(\mathcal{E}_{T}\right)=\lim _{\mathcal{E}_{A} \rightarrow 0} \frac{\Delta j}{\mathcal{E}_{A}}=\frac{d j\left(\mathcal{E}_{T}\right)}{d \mathcal{E}_{T}} .
$$

The fluctuation-induced tunneling conductivity $\sigma$ of the junction is then obtained by thermal averaging $\Sigma\left(\mathscr{E}_{T}\right)$ :

$$
\begin{aligned}
\sigma & =\int_{0}^{\infty} P\left(\mathcal{E}_{T}\right) \Sigma\left(\mathcal{E}_{T}\right) d \mathcal{E}_{T} \\
& =\int_{0}^{\infty} P\left(\mathcal{E}_{T}\right) \frac{d j\left(\mathcal{E}_{T}\right)}{d \mathcal{E}_{T}} d \mathcal{E}_{T} .
\end{aligned}
$$

At large applied field, the conductivity of the junction is expected to become non-ohmic. The behavior of the current density as a function of applied field in the high-field regime can be obtained by neglecting the small flow of electrons counter to the applied field and thermal averaging the forward current. Since the current along the appliedfield direction consists of $\frac{1}{2} j\left(\mathcal{E}_{A}-\mathcal{E}_{T}\right)$ (for $\left.\mathcal{E}_{T}<\mathcal{E}_{A}\right)$ and $\frac{1}{2} j\left(\mathcal{E}_{A}+\mathcal{E}_{T}\right)$, it follows that

$$
\begin{aligned}
j_{H}=\frac{1}{2}\left[\int_{0}^{\infty} j\left(\mathcal{E}_{A}+\mathcal{E}_{T}\right) P\left(\mathcal{E}_{T}\right) d \mathcal{E}_{T}+\int_{0}^{\mathcal{E}_{A}}\right. & j\left(\mathcal{E}_{A}-\mathcal{E}_{T}\right) \\
& \left.\times P\left(\mathcal{E}_{T}\right) d \mathcal{E}_{T}\right],
\end{aligned}
$$

where $j_{H}$ stands for the current density at high field.

In addition to modifying the tunneling probabilities, voltage fluctuations also have a direct effect on the parallel conduction mechanism of thermal activation over the potential barrier. However, as shown in Appendix A, thermal activation is negligible at low temperatures and gives behavior indistinguishable from that of fluctuation-induced tunneling at high temperatures. Therefore, the consideration of fluctuation-induced tunneling alone is sufficient to account for the conduction characteristics of the junction.

In order to further explore the temperature and the field dependences of the tunneling conductivity, it is clear that an explicit expression for the fielddependent tunneling current is necessary. The following subsections are devoted to the calculation of $j(\mathcal{E})$ and the evaluation of integrals appearing in Eqs. (6) and (7).

\section{A. Field-dependent tunneling current}

The starting point of our tunneling current calculation is the image-force corrected rectangular barrier ${ }^{11-14}$ as illustrated in Fig. 3. The imageforce correction removes the unphysical infinite fields implicitly implied by the rectangular barrier and makes the potential smoothly varying. Furth-

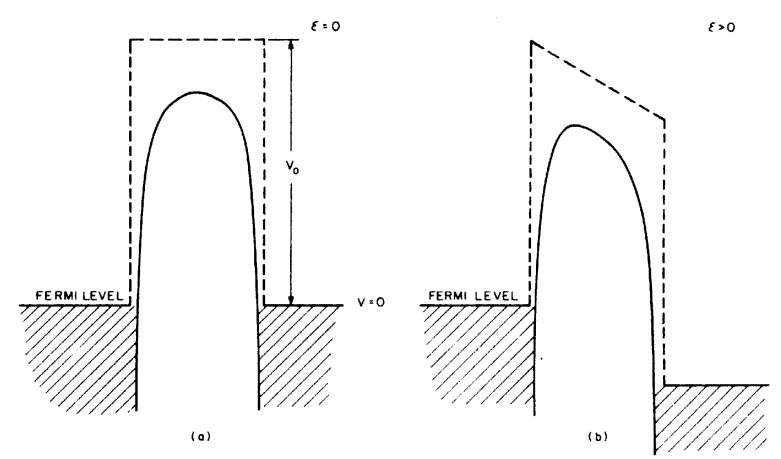

FIG. 3. The image-force corrected rectangular barrier in the absence or presence of an electric field $\mathcal{E}$. The dashed lines denote the rectangular barrier without the image potential. The solid lines are calculated from Eq. (8) with $\lambda=0.05$. 
ermore, the one additional parameter provided by the image-force correction enables us to consider a wide variety of barrier shapes, varying from nearly rectangular to nearly parabolic, upon which the effects of voltage fluctuations can be examined. As shown by Simmons, ${ }^{15}$ the shape of the potential in the presence of an electric field can be accurately expressed as

$$
V(u, \overline{\mathcal{E}})=V_{0}[1-\lambda / u(1-u)-\overline{\mathcal{E}} u],
$$

where $u=x / w$ is the reduced spatial variable, $x$ the distance from the left surface of the junction, $V_{0}$ is height of the rectangular potential barrier in the absence of image-force correction, $\overline{\mathcal{E}}=\mathcal{E} e w /$ $V_{0}$ is the electric field expressed in units of $\mathcal{E}_{V}$ $=V_{0} / e w$,

$$
\lambda=0.795 e^{2} / 4 w K V_{0}
$$

is a dimensionless parameter governing the amount of image-force correction and the resultant barrier shape, $e$ is the electronic charge, and $K$ is the dielectric constant of the insulating barrier. In Eq. (8) the zero of the potential is defined by the Fermi level of the conducting region. The potential $V(u, \overline{\mathcal{E}})$ is a peaked function of $u$. Its maximum, denoted by $V_{m}=V\left(u^{*}, \overline{\mathcal{E}}\right)$, where $u^{*}$ satis fies the condition $(\partial V / \partial u)_{u_{-} u^{*}}=0$, is a decreasing function of $\overline{\mathcal{E}}$. For a given $\lambda$, there is a value $\overline{\mathcal{E}}=\overline{\mathcal{E}}_{0}$ at which $V_{m}=0$. In Fig. $4, \overline{\mathcal{E}}_{0}$ is plotted as a function of $\lambda$. It should be noted that at $\lambda=\frac{1}{4}$, $V_{m}=0$, with $\overline{\mathcal{E}}=0$. Since the concept of a tunneling barrier becomes suspect when $V_{m} \leqslant 0$ even in the absence of any electric field, it is reasonable to signify $\frac{1}{4}$ as the maximum allowed value of $\lambda$. In terms of $\overline{\mathcal{E}}_{0}$, a new dimensionless field parameter can be defined as $\epsilon=\overline{\mathcal{E}} / \overline{\mathcal{E}}_{0}$. The advantage of using $\epsilon$ to characterize the field is that $\epsilon=1$ marks the point below which $V_{m} \geqslant 0$ and above which $V_{m} \leqslant 0$. As will be shown below, tunneling characteristics for $\epsilon \leqslant 1$ are drastically different from those for $\epsilon \geqslant 1$.

The tunneling current density $j(\epsilon)$ for a junction can be written as ${ }^{16}$

$$
\begin{aligned}
j(\epsilon)=\frac{4 \pi e}{\hbar} \int_{-\infty}^{\infty} d E D(E, \epsilon) \int \frac{d^{2} k_{\|}}{(2 \pi)^{2}}[ & f\left(E+E_{\|}\right)-f\left(E+E_{\|}\right. \\
& \left.\left.+e \epsilon \mathcal{E}_{0} w\right)\right] .
\end{aligned}
$$

Here $\hbar$ is the Planck's constant, $E$ is the energy of the electron in the direction of tunneling ( $x$ direction), $k_{\|}$is the wave vector of the electron parallel to the junction surface, $E_{\|}=\hbar^{2} k_{11}^{2} / 2 m, m$ being the effective mass of the electron, $\mathcal{E}_{0}=\overline{\mathcal{E}}_{0} \mathcal{E}_{V}$, and $f(E)=1 /[1+\exp (E / k T)]$ is the Fermi function. In the WKB approximation, the barrier transmission factor $D(E, \epsilon)$ is given ${ }^{17-19}$ by

$$
D(E, \epsilon)=\{1+\exp [F(E, \epsilon)]\}^{-1}, \quad E \leqslant V_{m},
$$

where

$$
F(E, \epsilon)=2 \chi w \int_{u_{1}}^{u_{2}}\left(\frac{V(u, \epsilon)-E}{V_{0}}\right)^{1 / 2} d u,
$$

$\chi=\left(2 m V_{0} / \hbar^{2}\right)^{1 / 2}$ is the tunneling constant, and $u_{1}$, $u_{2}\left(\geqslant u_{1}\right)$ are the zeros of the integrand. When $E$ $\geqslant V_{m}$, the WKB approximation also provides a transmission factor ${ }^{17-19}$ which connects smoothly to $D(E, \epsilon)$ given by Eq. (11) and which asymptotically approaches the value 1 at large $E$. However, it was shown ${ }^{20}$ that writing $D(E, \epsilon)$ in the form

$$
D(E, \epsilon)= \begin{cases}\exp [-F(E, \epsilon)], & E \leqslant V_{m}, \\ 1, & E>V_{m}\end{cases}
$$

introduces very little error. Since Eq. (13) is mathematically much simpler than the exact WKB expression, in the following this approximate form for $D(E, \epsilon)$ will be used.

The integral over $k_{\|}$in Eq. (10) can be simply performed, yielding

$$
j(\epsilon)=\frac{m e}{8 \pi^{2} \hbar^{3}} \int_{-\infty}^{\infty} d E D(E, \epsilon)\left[k T \ln \left(\frac{1+\exp (-E / k T)}{1+\exp \left[-\left(E+e \epsilon \mathcal{E}_{0} w\right) / k T\right]}\right)\right]
$$

The temperature dependence of the function $k T \ln [1+\exp (-E / k T)]$ arises from the smearing of the Fermi level at finite temperatures. Since $D(E, \epsilon)$ is an exponentially increasing function of $E$, for those electrons thermally activated from $E<0$ to $E>0$ the tunneling probability would be greatly enhanced. However, this increase in the tunneling probability has to be weighed against the decreased occupation probability for $E>0$. In Appendix $B$ it is shown that as long as tunneling is the dominant conduction mechanism (as opposed to thermal activation over the barrier) tunneling by electrons in the exponential tail above the Fermi level (thermally activated tunneling) is always negligible as compared to tunneling at the Fermi level. That is, the effect of Fermi level smearing can be ignored. Therefore, for the present calculation the low-temperature approximation of sharp Fermi level is adopted ${ }^{21}$ :

$$
k T \ln [1+\exp (-E / k T)] \cong\left\{\begin{array}{cc}
0, & E>0, \\
-E, & E<0 .
\end{array}\right.
$$




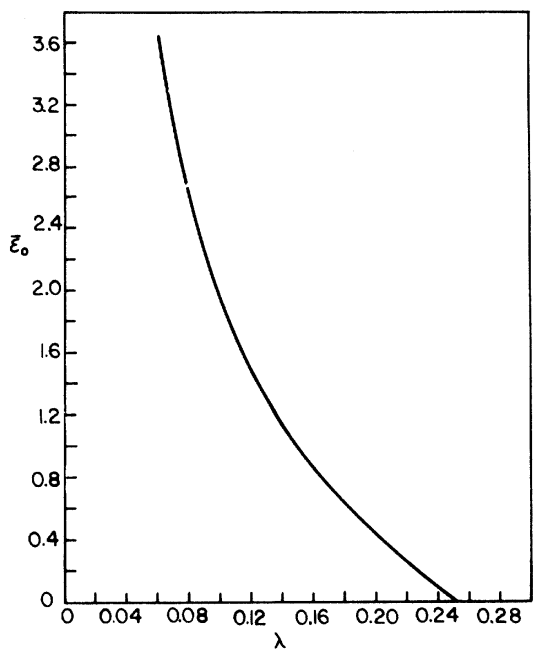

FIG. 4. Variation of $\overline{\mathcal{E}}_{0}$ as a function of $\lambda$.

A similar approximation for the factor $k T \ln \left\{1+\exp \left[-\left(E+e \in \mathcal{E}_{0} w\right) / k T\right]\right\}$ results in

$$
\begin{aligned}
& j(\epsilon)=\frac{m e}{8 \pi^{2} \hbar^{3}} \int_{-\infty}^{0} d E D(E, \epsilon) \Theta(E), \\
& \Theta(E)=\left\{\begin{array}{cc}
0, & E>0 \\
-E, & -e \epsilon \mathcal{E}_{0} w<E<0 \\
e \in \mathcal{E}_{0} w, & E<-e \epsilon \mathcal{E}_{0} w .
\end{array}\right.
\end{aligned}
$$

For $V_{m}>0(\epsilon<1)$, it is observed that the barrier transmission probability decreases rapidly below $E=0$. Therefore, most of the tunneling occurs in the vicinity of $E=0$, and we can approximate $F(E, \epsilon)$ by the first two terms of the power series expansion about $E=0$ :

$$
\begin{aligned}
& F(E, \epsilon) \cong 2 \chi w \xi(\epsilon)-\frac{2 \chi w}{V_{0}} \eta_{0}(\epsilon) E, \\
& \xi(\epsilon)=\int_{u_{3}}^{u_{4}}\left(1-\frac{\lambda}{u(1-u)}-\epsilon \overline{\mathcal{E}}_{0} u\right)^{1 / 2} d u, \\
& \eta_{0}(\epsilon)=\frac{1}{2} \int_{u_{3}}^{u_{4}} \frac{d u}{\left(1-\frac{\lambda}{u(1-u)}-\epsilon \overline{\mathcal{E}}_{0} u\right)^{1 / 2}} .
\end{aligned}
$$

Here $u_{3}, u_{4}\left(>u_{3}\right)$ denotes the two zeros of $V(u, \epsilon)$, and $\eta_{0}(\epsilon)$ is obtained from $\partial F(E, \epsilon) /\left.\partial E\right|_{E \neq 0}$ by noting that the terms containing the derivatives of $u_{1}$ and $u_{2}$ with respect to $E$ vanish, because the integrand of Eq. (12) is zero when evaluated at $u_{1}$ and $u_{2}$. Substitution of Eqs. (17) and (13) into Eq. (16) yields

$$
\begin{aligned}
j(\epsilon)= & \frac{m e}{8 \pi^{2} \hbar^{3}} \exp [-2 \chi w \xi(\epsilon)] \\
& \times \int_{-\infty}^{0} d E \Theta(E) \exp \left[\frac{2 \chi w}{V_{0}} \eta_{0}(\epsilon) E\right], \quad \epsilon<1 .
\end{aligned}
$$

In Eq. (18) the presence of the factor $\exp \left[2 \chi w \eta_{0}(\epsilon) E / V_{0}\right]$ makes the value of the integral only sensitive to the form of $\Theta(E)$ at $E \simeq 0$. Therefore, we can let $\Theta(E) \simeq-E$ for $-\infty<E<0$ without incurring much error. The resulting integral gives

$$
j(\epsilon)=\frac{m e}{8 \pi^{2} \hbar^{3}}\left(\frac{V_{0}}{2 \chi w \eta_{0}(\epsilon)}\right)^{2} \exp [-2 \chi w \xi(\epsilon)], \quad \epsilon<1 .
$$

For $V_{m}<0(\epsilon>1)$, Eq. (16) can be written as

$$
\begin{gathered}
j(\epsilon)=\frac{m e}{8 \pi^{2} \hbar^{3}}\left[\int_{-\infty}^{V_{m}} d E \exp [-F(E, \epsilon)] \Theta(E)\right. \\
\left.+\int_{V_{m}}^{0} d E \Theta(E)\right] .
\end{gathered}
$$

By expanding $F(E, \epsilon)$ in a power series about $V_{m}$, we obtain

$$
F(E, \epsilon) \cong-\frac{2 \chi w}{V_{0}} \eta_{1}(\epsilon)\left(E-V_{m}\right),
$$

where

$$
\begin{aligned}
\eta_{1}(\epsilon) & =-\left.\frac{V_{0}}{2 \chi w} \frac{\partial F(E, \epsilon)}{\partial E}\right|_{E=V_{m}} \\
& =\frac{\pi}{2\left(\epsilon \overline{\mathcal{E}}_{0}\right)^{1 / 2}}\left(\frac{u^{*}\left(1-u^{*}\right)\left(1-2 u^{*}\right)}{1-3 u^{*}\left(1-u^{*}\right)}\right)^{1 / 2},
\end{aligned}
$$

and $u^{*}$, denoting the location of $V_{m}$, satisfies the equation

$$
0=\left.\frac{\partial V(u, \epsilon)}{\partial u}\right|_{u=u *}=\frac{1-2 u^{*}}{\left(u^{*}\right)^{2}\left(1-u^{*}\right)^{2}}-\frac{\epsilon \overline{\mathcal{E}}_{0}}{\lambda} .
$$

Since the relation $\left|V_{m}\right|<e \in \mathcal{E}_{0} w$ always holds for $V_{m}<0$ [see Eq. (24) below], we can effectively replace $\Theta(E)$ in Eq. (20) by its value in the interval $-e \in \mathcal{E}_{0} w<E<0$. That is,

$$
\begin{aligned}
j(\epsilon) & =\frac{m e}{8 \pi^{2} \hbar^{3}}\left[\frac{V_{m}^{2}}{2}+\int_{-\infty}^{V_{m}} d E \exp \left(\frac{2 \chi w}{V_{0}} \eta_{1}(\epsilon)\left(E-V_{m}\right)\right)(-E)\right] \\
& =\frac{m e}{8 \pi^{2} \hbar^{3}}\left[\frac{V_{m}^{2}}{2}+\left(\frac{V_{0}}{2 \chi w \eta_{1}(\epsilon)}\right)^{2}\left(1-\frac{2 \chi w}{V_{0}} \eta_{1}(\epsilon) V_{m}\right)\right], \quad \epsilon>1 .
\end{aligned}
$$

From numerical calculations it is found that the variation of $V_{m}$ (for $V_{m}<0$ ) as a function of $\epsilon$ can be approximated as

$$
V_{m} \cong 2.2 \lambda V_{0} \overline{\mathcal{E}}_{0}(1-\epsilon) \text {. }
$$


Substituting Eq. (24) into Eq. (23) and combining the results of $j(\epsilon)$ for $\epsilon>1$ and $\epsilon<1$, we get

$$
\begin{aligned}
& j(\epsilon)= \begin{cases}j_{0}(\epsilon) \exp [-2 \chi w \xi(\epsilon)], & \epsilon \leqslant 1 \\
j_{1}(\epsilon) & \epsilon>1,\end{cases} \\
& j_{0}(\epsilon)=\frac{j_{00}}{\eta_{0}^{2}(\epsilon)}, \epsilon \leqslant 1, \\
& j_{1}(\epsilon)=j_{00}\left(\frac{1}{\eta_{1}^{2}(\epsilon)}+9.68\left(\lambda \chi w \overline{\mathcal{E}}_{0}\right)^{2}(1-\epsilon)^{2}-4.4\left(\lambda \times w \overline{\mathcal{E}}_{0}\right) \frac{1-\epsilon}{\eta_{1}(\epsilon)}\right), \quad \epsilon>1, \\
& j_{00}=\frac{\pi m e V_{0}^{2}}{4 \chi^{2} w^{2} h^{3}} .
\end{aligned}
$$

Here $h=2 \pi \hbar$, and the value of $j(\epsilon)$ is noted to be continuous at $\epsilon=1$, since from the definitions of $\eta_{0}$ and $\eta_{1}$ the relation $\eta_{0}(1)=\eta_{1}(1)$ holds. From $j(\epsilon)$ we can also obtain an expression for $\Sigma(\epsilon)$ :

$$
\begin{aligned}
\Sigma(\epsilon) & =\frac{1}{\mathcal{E}_{0}} \frac{d j(\epsilon)}{d \epsilon} \\
& = \begin{cases}\Sigma_{0}(\epsilon) \exp [-2 \chi w \xi(\epsilon)], & \epsilon \leqslant 1 \\
\Sigma_{1}(\epsilon), & \epsilon>1,\end{cases}
\end{aligned}
$$

where

$$
\begin{aligned}
& \Sigma_{0}(\epsilon)=-\Sigma_{00} \frac{1}{\eta_{0}^{2}(\epsilon)}\left[\frac{1}{\eta_{0}(\epsilon)} \frac{d \eta_{0}(\epsilon)}{d \epsilon}+\chi w \frac{d \xi(\epsilon)}{d \epsilon}\right], \quad \epsilon \leqslant 1, \\
& \Sigma_{1}(\epsilon)=\Sigma_{00}\left[\frac{2.2 \lambda \chi w \overline{\mathcal{E}}_{0}}{\eta_{1}(\epsilon)}-\frac{1}{\eta_{1}^{3}(\epsilon)} \frac{d \eta_{1}(\epsilon)}{d \epsilon}+\left(\frac{2.2 \lambda \chi w \overline{\mathcal{E}}_{0}}{\eta_{1}^{2}(\epsilon)} \frac{d \eta_{1}(\epsilon)}{d \epsilon}-9.68\left(\lambda \chi w \overline{\mathcal{E}}_{0}\right)^{2}\right)(1-\epsilon)\right], \quad \epsilon>1, \\
& \Sigma_{00}=2 j_{00} / \mathcal{E}_{0} .
\end{aligned}
$$

Explicit numerical evaluations of $\Sigma_{0}(\epsilon)$ and $\Sigma_{1}(\epsilon)$ in Appendix $\mathrm{C}$ show them to be slowly varying functions of $\epsilon$. Therefore, Eq. (26a) states the intuitive result that the differential conductivity of the junction increases as an exponential function of the field for $\epsilon \leqslant 1$ but only varies slowly when $\epsilon>1$. Since $\Sigma_{1}(1) \neq \Sigma_{0}(1)$ (see Appendix C), $\Sigma(\epsilon)$ has a sudden change of value at $\epsilon=1$. This discontinuity is an artifact of using the form of $D(E, \epsilon)$ given by Eq. (13), which approximates the variation of $D(E, \epsilon)$ at $E=V_{m}$ by an abrupt slope change.

\section{B. Fluctuation-induced tunneling conductivity}

The calculation of the junction conductivity $\sigma$ involves thermal averaging $\Sigma(\epsilon)$ as expressed by Eq. (6):

$$
\begin{aligned}
\sigma=\left(\frac{4 T_{1}}{\pi T}\right)^{1 / 2} & {\left[\int_{0}^{1} d \epsilon_{T} \Sigma_{0}\left(\epsilon_{T}\right) \exp \left(-\frac{T_{1}}{T} \epsilon_{T}^{2}-\frac{T_{1}}{T_{0}} \varphi\left(\epsilon_{T}\right)\right)\right.} \\
& \left.+\int_{1}^{\infty} d \epsilon_{T} \Sigma_{1}\left(\epsilon_{T}\right) \exp \left(-\frac{T_{1}}{T} \epsilon_{T}^{2}\right)\right] . \quad \text { (27a) }
\end{aligned}
$$

The new parameters $T_{1}$ and $T_{0}$ are defined as

$$
T_{1}=a \mathcal{E}_{0}^{2} / k
$$

$$
T_{0}=T_{1} / 2 \chi w \xi(0),
$$

$\epsilon_{T}=\mathcal{E}_{T} / \mathcal{E}_{0}$, and the function $\varphi(\epsilon)$ is given by

$$
\varphi(\epsilon)=\xi(\epsilon) / \xi(0) \text {. }
$$

$\varphi(\epsilon)$ has the property that $\varphi(0)=1$ and $\varphi(1)=0$. The functions $-T_{1} \varphi\left(\epsilon_{T}\right) / T_{0}$ and $-T_{1} \epsilon_{T}^{2} / T$ are plotted in Fig. 5. It is seen that at small values of $\epsilon_{T}$, the tunneling probability increases faster with increasing field than the fluctuation probability decreases. However, at larger values of $\epsilon_{T}$ the reverse becomes the case. As a result, the sum of the two factors, denoted by the dashed line, goes through a maximum at some value $\epsilon_{T}$ $=\epsilon^{*}$. Since the existence of a maximum in the exponent implies that the integrand of the first integral in Eq. (27a) is sharply peaked, it is expected that the temperature dependence of $\sigma$ would be dominated by the variation of $\epsilon^{*}$. In anticipation of this fact, we write

$$
\sigma=\sigma_{0} \exp \left[-\frac{T_{1}}{T}\left(\epsilon^{*}\right)^{2}-\frac{T_{1}}{T_{0}} \varphi\left(\epsilon^{*}\right)\right]
$$

where $\sigma_{0}$ is defined as 


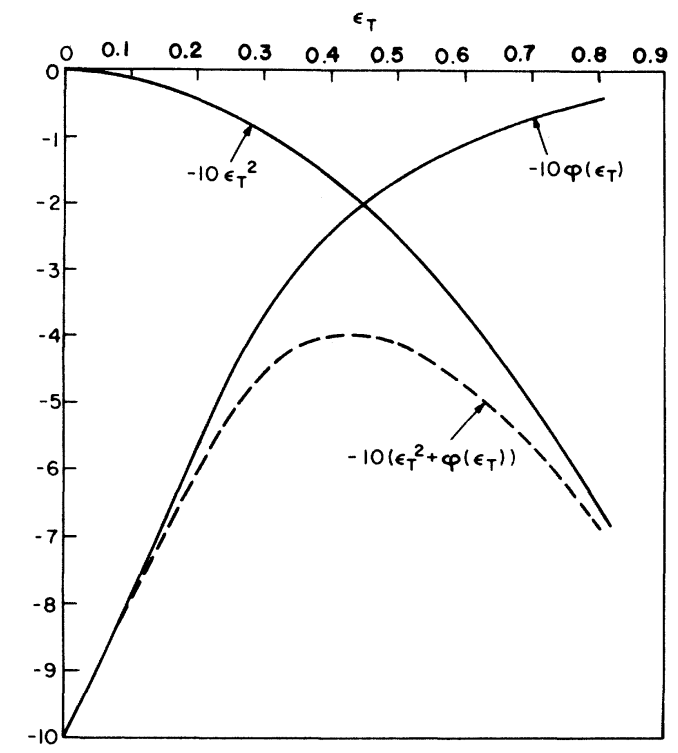

FIG. 5. The increase in the tunneling contribution as a function of $\epsilon_{T}$, expressed by $-10 \varphi\left(\epsilon_{T}\right)$, is opposed by the decrease of the fluctuation probability, expressed by $-10 \epsilon_{T}^{2}$. The maximum that obtains from the sum of these two terms represents the best compromise between the two tendencies. The function $-10 \varphi\left(\epsilon_{T}\right)$ shown in the figure is calculated with $\lambda=0.05$.

$$
\begin{aligned}
\sigma_{0}= & \exp \left[+\frac{T_{1}}{T}\left(\epsilon^{*}\right)^{2}+\frac{T_{1}}{T_{0}} \varphi\left(\epsilon^{*}\right)\right]\left(\frac{4 T_{1}}{\pi T}\right)^{1 / 2} \\
\times & {\left[\int_{0}^{1} d \epsilon_{T} \Sigma_{0}\left(\epsilon_{T}\right) \exp \left(-\frac{T_{1}}{T} \epsilon_{T}^{2}-\frac{T_{1}}{T_{0}} \varphi\left(\epsilon_{T}\right)\right)\right.} \\
& \left.+\int_{0}^{\infty} d \epsilon_{T} \Sigma_{1}\left(\epsilon_{T}\right) \exp \left(-\frac{T_{1}}{T} \epsilon_{T}^{2}\right)\right],
\end{aligned}
$$

and $\epsilon^{*}$ is the location of the maximum value of $-\left[\left(T_{1} / T\right) \epsilon^{2}+\left(T_{1} / T_{0}\right) \varphi(\epsilon)\right]$ in the interval $0 \leqslant \epsilon \leqslant 1$. What we have done in Eqs. (28) and (29) is simply to factor out the quantity $\exp \left[-T_{1}\left(\epsilon^{*}\right)^{2} / T-T_{1} \varphi\left(\epsilon^{*}\right) /\right.$ $T_{0}$ ] from $\sigma$, Eq. (27a), and call the rest $\sigma_{0}$. If our expectation is correct, $\sigma_{0}$ should be a slowly varying function of $T$ as compared to the exponential term. In Appendix $\mathrm{C}$ explicit numerical evaluation of Eq. (29) shows this indeed to be the case. Therefore, in Eq. (28) $\sigma_{0}$ can be treated as a constant.

The high- and the low-temperature behaviors of $\sigma$ can be immediately deduced from Eq. (28). Since $\epsilon^{*}$ decreases with decreasing temperature, at low temperatures $\epsilon^{*}$ is small and we can write $-\varphi(\epsilon)$ $\simeq S \epsilon$, where $S$ is the slope of $\varphi(\epsilon)$ at $\epsilon=0$. In this case $\epsilon^{*}=\left(S / 2 T_{0}\right) T$ and $-\left(T_{1} / T\right)\left(\epsilon^{*}\right)^{2} \rightarrow 0$ as $T$ approaches 0 . Consequently, at very low temperatures $\sigma$ is temperature independent and reduces to the expected form $\sigma_{0} \exp [-2 \chi w \xi(0)]$ for simple elastic tunneling. As the temperature increases, $\epsilon^{*}$ also increases until it reaches the value 1 . Since $\epsilon^{*}$ is defined to lie within 0 and $1, \epsilon^{*}$ will stay at 1 as $T$ rises further. The limiting hightemperature behavior is therefore thermally activated, i.e., given by $\sigma_{0} \exp \left(-T_{1} / T\right)$. Of course, we should realize that the validity of $\sigma_{0} \exp \left(-T_{1} / T\right)$ as expressing a thermally activated characteristic relies on the fact that $\Sigma_{1}(\epsilon)$ is a nonexponential function of $\epsilon$, and as a result $\sigma_{0}$ varies only slowly at high temperatures as shown in Appendix C.

In the intermediate temperature range the calculation of $\epsilon^{*}$ has to be performed numerically. Since the value of $\epsilon^{*}$ is bound between 0 and 1 as discussed above, $\epsilon^{*}$ can be found by locating the position of the maximum value for the function $-T_{1} \epsilon^{2} / T-T_{1} \varphi(\epsilon) / T_{0}, 0<\epsilon<1$. This is done on a computer using the Fibonacci search routine. The results for $\ln \left(\rho / \rho_{0}\right)$, where $\rho=1 / \sigma$, are plotted in Fig. 6 as a function of $1 / T$ for fixed values of $T_{1}=50 \mathrm{~K}$ and $T_{0}=5 \mathrm{~K}$. We see that by changing the value of $\lambda$, which is equivalent to varying the barrier shape, one can generate a variety of temperature variations interpolating between the thermally activated behavior at high temperatures and temperature-independent tunneling at low temperatures. Generally speaking, barriers that are nearly rectangular in shape $(\operatorname{small} \lambda)$ yield curves of $\ln \left(\rho / \rho_{0}\right)$ which increase toward the low-temperature asymptote much more slowly than the more rounded barriers (large $\lambda$ ). For comparison, the curve for the parabolic barrier is also plotted. The relevant equation for the parabolic barrier approximation is given in the next section.

In the above discussion it is shown that the temperature dependence of the low-field conductivity $\sigma$ is essentially determined by the variation of $\epsilon^{*}$. At any fixed temperature, if the applied field $\epsilon_{A}$

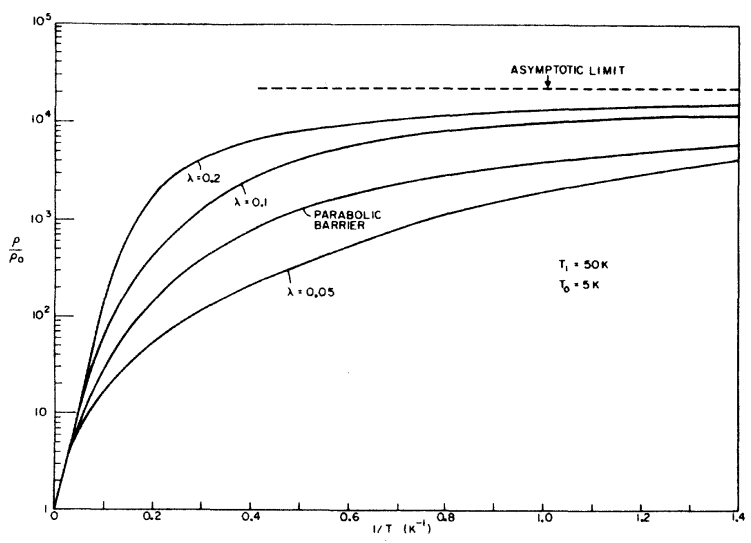

FIG. 6. Normalized fluctuation-induced resistivity as a function of $1 / T$ for various shapes of the tunneling barrier. All the curves are calculated with the same values of $T_{1}$ and $T_{0}$ given in the figure. 
$=\mathcal{E}_{A} / \mathcal{E}_{0}$ is comparable to or larger than $\epsilon^{*}$, it is expected that the junction conductivity would deviate drastically from that given by Eq. (29) due to the additional tunneling induced by $\epsilon_{A}$. In such a high-field regime the behavior of the tunneling current density $j_{H}$ is obtained by substituting Eq. (25) into Eq. (7),

$$
\begin{aligned}
j_{H}=\left(\frac{T_{1}}{\pi T}\right)^{1 / 2}\left\{\int_{0}^{\tau\left(\epsilon_{A}\right)} d \epsilon_{T} j_{0}\left(\epsilon_{A}+\epsilon_{T}\right)\right. \\
\quad \times \exp \left[-\frac{T_{1}}{T} \epsilon_{T}^{2}-\frac{T_{1}}{T_{0}} \varphi\left(\epsilon_{A}+\epsilon_{T}\right)\right] \\
+\int_{\tau\left(\epsilon_{A}\right)}^{\infty} d \epsilon_{T} j_{1}\left(\epsilon_{A}+\epsilon_{T}\right) \exp \left(-\frac{T_{1}}{T} \epsilon_{T}^{2}\right) \\
\left.+\int_{0}^{\epsilon_{A}} d \epsilon_{T} j\left(\epsilon_{A}-\epsilon_{T}\right) \exp \left(-\frac{T_{1}}{T} \epsilon_{T}^{2}\right)\right\} .
\end{aligned}
$$

Here the integration limit $\tau\left(\epsilon_{A}\right)$ is defined as

$$
\tau\left(\epsilon_{A}\right)= \begin{cases}1-\epsilon_{A}, & \epsilon_{A} \leqslant 1 \\ 0, & \epsilon_{A}>1\end{cases}
$$

Of the three integrals in Eq. (30a), the integrand of the first integral is sharply peaked. Similar to the case shown in Fig. 5 , the exponent $-\left(T_{1} \epsilon_{T}^{2} / T\right)$ $-T_{1} \varphi\left(\epsilon_{A}+\epsilon_{T}\right) / T_{0}$ also has a maximum at some value of $\epsilon_{T}$, denoted by $\epsilon^{+}$. By again repeating the arguments leading to the expression for $\sigma$, Eq. (28), we get

$$
j_{H} \cong j_{H}^{0} \exp \left[-\frac{T_{1}}{T}\left(\epsilon^{+}\right)^{2}-\frac{T_{1}}{T_{0}} \varphi\left(\epsilon_{A}+\epsilon^{+}\right)\right],
$$

where $j_{H}^{0}$ is taken to be a constant and $\epsilon^{+}$is the location of the maximum value of $-T_{1} \epsilon^{2} / T$ $-T_{1} \varphi\left(\epsilon_{A}+\epsilon\right) / T_{0}$ in the interval $0 \leqslant \epsilon \leqslant \tau\left(\epsilon_{A}\right)$. For definitiveness, we will set $j_{H}^{0}$ equal to the value of $j_{H}$ at $\epsilon_{A}=1$ when $T \rightarrow 0$. From Eq. (30a) this is easily evaluated as

$$
j_{H}^{0}=j_{00} / \eta_{0}^{2}(1) \text {. }
$$

The accuracy of the approximation expressed by Eqs. (31a) and (31b) is examined in Appendix C. Through comparison of $\boldsymbol{j}_{H}$ values evaluated from both Eq. (30) (by direct numerical integration) and Eq. (31), it is shown that at low temperatures Eq. (31) is (to within a multiplicative constant) descriptive of the actual $j_{H}$ field dependence for $\epsilon_{A}$ $<0.5$. However, at high temperatures or $\epsilon_{A}>0.75$ the approximation becomes less exact. Therefore, Eq. (31) should only be regarded as a lowtemperature approximation for $j_{H}$. Its use in fitting the data is expected to yield slightly distorted values for the parameters $\lambda, T_{1}$, and $T_{0}$. At high temperatures the full Eq. (30) has to be used.

Before leaving this section, it is observed that since the function $\varphi(\epsilon)$ is central to the calcula- tion of $\sigma$ and $j_{H}$, the numerical integration required for its evaluation [see Eqs. (17b) and (27d)] poses a rather serious inconvenience. In order to overcome this problem, we show in Appendix D that, to good accuracy, $\varphi(\epsilon)$ can be approximated by a rational function

$$
\varphi(\epsilon) \cong(1-\epsilon) /\left(1+\alpha \epsilon+\beta \epsilon^{2}\right) .
$$

The coefficients $\alpha$ and $\beta$ are determined by the requirement that the derivatives of $\varphi$ and its rational approximation agree at $\epsilon=0$ and $\epsilon=1$. Since these derivatives are functions of $\lambda$, it is found that to good accuracy,

$$
\begin{aligned}
& \alpha= 4.2837 \times 10^{-4}\left(\frac{1}{\lambda}-4\right)^{2} \\
&+4.8728 \times 10^{-2}\left(\frac{1}{\lambda}-4\right) \\
& \beta= \operatorname{coth}\left(\frac{2.6 \lambda}{1-4 \lambda}\right)-(1+\alpha) . \\
& \text { C. Parabolic-barrier approximation }
\end{aligned}
$$

The parabolic barrier, defined as

$$
V_{p}(u, \epsilon)=V_{0}\left[u(1-u)-\epsilon \overline{\mathcal{E}}_{0} u\right],
$$

has sometimes been used as an approximation to the image-forced corrected rectangular barrier. ${ }^{12,22}$ Such approximation is particularly accurate for $\lambda \cong 0.07$ and results in simple, closed expressions for $\sigma$ and $j_{H}$ as seen below.

In Eqs. (28) and (31a) the effect of barrier shape is noted to enter the picture only through the function $\varphi(\epsilon)$. For the parabolic barrier, $\varphi(\epsilon)=\xi(\epsilon) /$ $\xi(0)$ is given by

$$
\varphi(\epsilon)=\frac{\int_{0}^{1-\epsilon}[u(1-u)-\epsilon u]^{1 / 2} d u}{\int_{0}^{1}[u(1-u)]^{1 / 2} d u}=(1-\epsilon)^{2},
$$

where we have used the fact that $\overline{\mathcal{E}}_{0}=1$ and the roots of the integrand in the numerator occur at 0 and $(1-\epsilon)$. This explicit form of $\varphi(\epsilon)$ directly yields

$$
\begin{aligned}
& \epsilon^{*}=T /\left(T+T_{0}\right), \\
& \epsilon^{*}=\left(1-\epsilon_{A}\right) T /\left(T+T_{0}\right) .
\end{aligned}
$$

Substituting Eqs. (34) and (35) into Eqs. (28) and (31a) gives

$$
\begin{aligned}
& \sigma=\sigma_{0} \exp \left(-\frac{T_{1}}{T+T_{0}}\right), \\
& j_{H}=j_{H}^{0} \exp \left[-\frac{T_{1}}{T+T_{0}}\left(1-\epsilon_{A}\right)^{2}\right] .
\end{aligned}
$$

The behavior of $\rho(=1 / \sigma)$ expressed by Eq. (36a) is plotted in Fig. 6 with $T_{1}=50 \mathrm{~K}$ and $T_{0}=5 \mathrm{~K}$.

The physical meaning of $T_{0}$ and the effect of voltage fluctuations on the tunnel barrier are made 
more transparent by the closed form of Eq. (36). Since $T_{0}$ always appears as an additive constant to $T$, it can be viewed as the temperature above which the fluctuation effects become significant. At $T \ll T_{0}$ (or $\left.T_{0} \rightarrow \infty\right)$, Eqs. (36a) and (36b) are essentially temperature independent and reduce to $\sigma$ $\cong \sigma_{0} \exp (-\pi \chi w / 4), j_{H} \cong j_{H}^{0} \exp \left[-\pi \chi w\left(1-\epsilon_{A}\right)^{2} / 4\right]$, exactly the expressions expected for simple tunneling. Therefore, it can be said that simple elastic tunneling is just a special case of fluctuation-induced tunneling. When the temperature is increased from $T \ll T_{0}$ to $T \cong T_{0}$, the absolute magnitude of the exponents in the $\sigma$ and $j_{H}$ expressions are seen to decrease from their $T=0$ limiting values, implying that the tunnel barrier seen by the electrons is effectively reduced in height by the fluctuating voltages. Furthermore, the form of the exponents suggests that the most rapid change in the effective barrier height occurs when $T$ is on the same order as $T_{0}$. As will be seen in Sec. V, these theoretical predictions are verified with remarkable accuracy by experimental results.

\section{RANDOM NETWORK OF TUNNEL JUNCTIONS}

A disordered material necessarily contains a large number of tunnel junctions with diverse junction parameter values. As a result, the effects of voltage fluctuations are expected to vary from one junction to another. For example, there could be junctions with very small values of $w$ which imply, on the one hand, the quenching of voltage fluctuations by the associated large capacitances and, on the other hand, a greatly enhanced probability for simple elastic tunneling. However, no matter what the values of the junction parameters might be, the discussion of the last section shows that the behavior of each junction is expressible in terms of the single junction characteristics with appropriate values of $T_{1}$ and $T_{0}$ (simple elastic tunneling being just a special case of fluctuation-induced tunneling). Therefore, as far as conductivity is concerned, the disordered material in question may be regarded as random resistor network in which the resistors are identified as the tunnel junctions with distributions in the values of $T_{1}$ and $T_{0}$, and the nodes are identified as the conducting pathways connecting the different junctions.

The conductivity of a random resistor network can be calculated by the self-consistent effectivemedium theory as developed by Kirkpatrick ${ }^{23}$ and Bernasconi. ${ }^{24}$ In this method the average effect of random conductances $\sigma_{i}$ is treated by replacing all conductances by a single value $\sigma_{m}$ which is then related to the network conductivity by a proportionality factor. $\sigma_{m}$ is determined by the equation

$$
\int d \bar{\sigma} \frac{D(\bar{\sigma})}{\bar{\sigma}+\left(\frac{1}{2} z-1\right) \bar{\sigma}_{m}}=\frac{2}{z \bar{\sigma}_{m}} .
$$

Here $\bar{\sigma}=\sigma / \sigma_{0}, \bar{\sigma}_{m}=\sigma_{m} / \sigma_{0}, \sigma_{0}$ is treated as a multiplicative constant, $D(\bar{\sigma})$ is the distribution function of the conductances, $z$ is the average number of resistors emanating from a node, and the integral is over all possible values of the conductances. Since, at fixed $T$, the variation in $\bar{\sigma}$ is directly linked to distributions in the values of $T_{1}$ and $T_{0}$, we can write

$$
D(\bar{\sigma}) d \bar{\sigma}=\left[\int_{0}^{\infty} d T_{1} D\left(T_{1}\right) D\left(T_{0}\left(\bar{\sigma}, T_{1}\right)\right)\left|\frac{\partial T_{0}}{\partial \bar{\sigma}}\right|\right] d \bar{\sigma},
$$

where $D\left(T_{1}\right), D\left(T_{0}\right)$ are the distributions of $T_{1}$ and $T_{0}$, and we have regarded $T_{0}$ as a dependent variable determined by $\bar{\sigma}$ and $T_{1}$. From Eq. (38) it is easy to see that a change of variable from $\bar{\sigma}$ to $T_{0}$ makes

$$
\int d \bar{\sigma} D(\bar{\sigma})-\int_{0}^{\infty} \int_{0}^{\infty} d T_{1} d T_{0} D\left(T_{1}\right) D\left(T_{0}\right),
$$

which means Eq. (37) can be rewritten as

$$
\int_{0}^{\infty} \int_{0}^{\infty} d T_{1} d T_{0} \frac{D\left(T_{1}\right) D\left(T_{0}\right)}{\bar{\sigma}+\left(\frac{1}{2} z-1\right) \bar{\sigma}_{m}}=\frac{2}{z \bar{\sigma}_{m}} .
$$

For the purpose of explicit calculation in this paper, the functions $D\left(T_{1}\right)$ and $D\left(T_{0}\right)$ are taken to be $\log$-normal distributions ${ }^{25}$

$$
D(y)=\frac{1}{2 \pi} \frac{1}{\ln \mu} \exp \left[-\frac{(\ln y)^{2}}{2(\ln \mu)^{2}}\right] \frac{1}{y},
$$

where $y=T_{1} / T_{1}^{m}$ or $T_{0} / T_{0}^{m}, T_{1}^{m}$ and $T_{0}^{m}$ are, respectively, the median values of the distributions $D\left(T_{1}\right)$ and $D\left(T_{0}\right)$, and $\mu$ is the parameter which controls the width of the distribution. As shown in Fig. 7, $D(y)$ is a peaked function of $y$ which vanishes at $y=0$ and $y=\infty$. When $\mu-1$, the function $D(y)$ becomes the delta function, and in that case

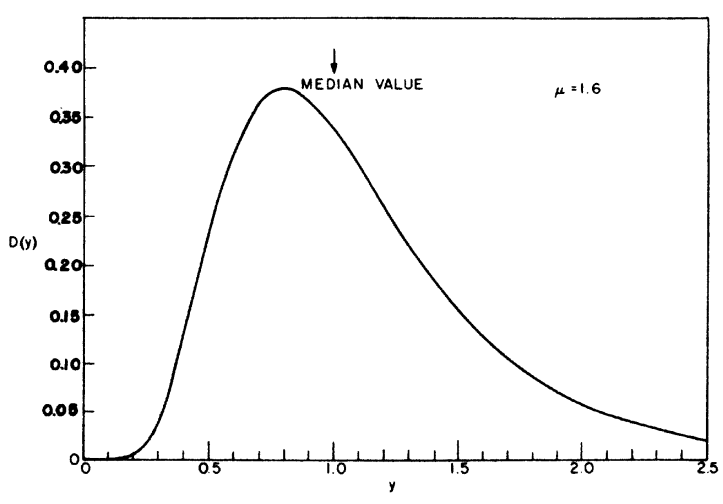

FIG. 7. The log-normal distribution calculated from Eq. (40). 
it is easily verified that Eq. (39) yields

$$
\bar{\sigma}_{m}=\bar{\sigma}\left(T_{1}^{m}, T_{0}^{m}\right),
$$

that is, the conductivity of the network is given by the single junction behavior. When $\mu>1$, Eq. (39) has to be solved numerically. Bu substituting Eq. (28) into Eq. (39), we get an integral relation in which $T, T_{1}^{m}, T_{0}^{m}, \mu$, and $z$ are the inputs and $\bar{\sigma}_{m}$ is the unknown. In Sec. V the solution of Eq. (39) will be directly compared with the experimental results.

In the high-field regime, we can define a highfield conductivity, $\sigma_{H}=j_{H} / \mathcal{E}_{A}$, for each junction. By replacing $\sigma$ with $\sigma_{H}$ in Eq. (39), the network conductance at any applied (macroscopic average) field (assumed to be related to $\mathcal{E}_{A}$ by a constant factor $M$ as discussed before) can be obtained in exactly the same fashion as for the low-field case.

\section{APPLICATION TO DISORDERED SYSTEMS}

\section{A. Carbon-polyvinylchloride (C-PVC) composites}

C-PVC is a conductor-insulator composite ${ }^{3,26}$ consisting of carbon particles embedded in the insulating PVC matrix. Three types of carbon were used $^{27}$ : (1) Ketjenblack ${ }^{\circledR}$ is a pigment composed of hollow spheres approximately $350 \AA$ in diameter with 10-15 ̊-thick walls; (2) Columbia carbon SA40-220 is similar to Ketjenblack but has a sphere diameter of $140 \AA$; (3) Mogul-L $L^{\circledR}$ carbon is a pigment containing carbon in the form of solid spheres with a diameter of about $200 \AA$ Electron micrograph studies show the carbons to possess a high degree of connectivity, ${ }^{28}$ that is, a carbon network is usually observed which is composed of touching or nearly touching micron-sized aggregates of graphitic spheres. Of the three types of carbon mentioned above, Mogul-L showed some difference from the other two in exhibiting less of the tendency to form clumps and chains. As a result, samples with Mogul-L carbon generally show much higher resistivity than those with Ketjenblack or SA40-220.

Electrical conductivity of C-PVC composites results from percolation of electrons in the carbon networks. When the concentration of the carbon is large, the material exhibits graphitic conductivity, ${ }^{29}$ indicating that the conduction network is continuous. As the concentration is decreased, conduction is dominated by electron tunneling across small barriers separating the conducting pathways. In Fig. 8 the temperature dependence of resistivity is shown for four samples. The solid lines are the theoretical fits to the data using Eqs. (28) and (39) with $\lambda=0.07, z=6$, and $\mu=1$, i.e., single-junction behavior. The values of $T_{0}$ and $T_{1}$ are labeled beside each curve. The dashed

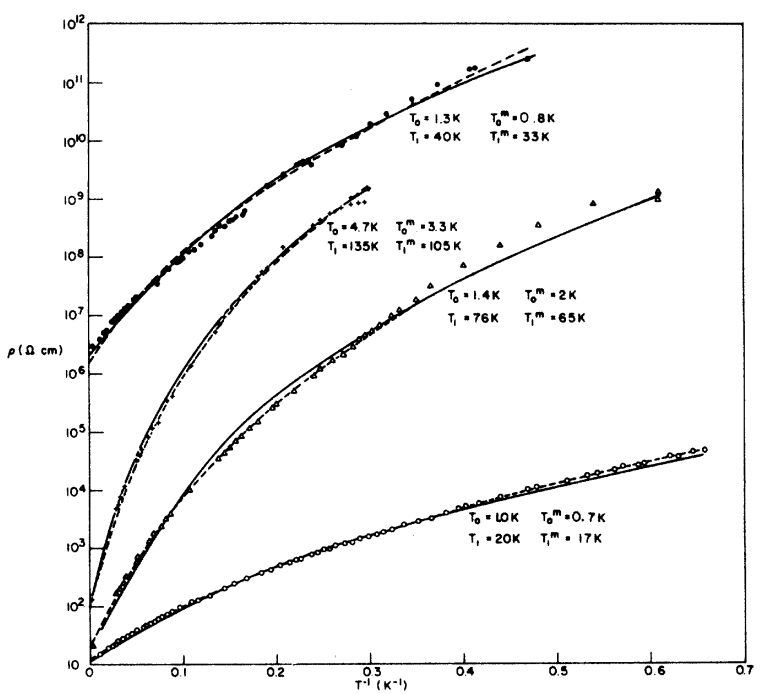

FIG. 8. Temperature dependence of the resistivity in carbon-PVC composites. Solid circles, Mogul-L, 45wt. \%; pluses, SA40-220, 15-wt. \%; triangles, SA40-220, 20 -wt. \%; open circles, Ketjenblack, 16-wt.\%. Solid curves are calculated from Eqs. (28) and (39) with $\mu=1$ and values of $T_{1}$ and $T_{0}$ given in the figure. Dashed lines are calculated from the same equations with $\mu=1.6$ and values of $T_{1}^{m}$ and $T_{0}^{m}$ labeled beside each curve.

lines are the theoretical fits using the same equations but now with $\mu=1.6$, i.e., a distribution of junction parameters. The values of $\rho$ in this case are obtained numerically from Eq. (39) by using the Gaussian integration routines to evaluate the double integral and the Newton-Ralphson's method to locate the value of $\bar{\sigma}_{m}(\propto 1 / \rho)$ for each $T$. The median values of the distributions used in the calculation, $T_{1}^{m}$ and $T_{0}^{m}$. are also given in the figure. It is seen that equally good agreements between the theory and the experimental data can be achieved by using either the single-junction char acteristic $(\mu=1)$ or a distribution of junction parameters $(\mu>1)$. However, the median values of $T_{1}$ and $T_{0}$ obtained from the fits are dependent on the width of the junction parameter distributions. Since at present there are no data on the $T_{1}$ and $T_{0}$ distributions, in the following the experimental results will be directly compared with the singlejunction characteristics for simplicity. Of course, the values of $T_{0}$ and $T_{1}$ which result from such fitting of the experimental data should be interpreted as indicative of the median values for $T_{0}$ and $T_{1}$ rather than as the parameters for a single junction.

It should be remarked here that the same data shown in Fig. 8 have been treated in Ref. 3 using the parabolic barrier approximation. Due to the fact that the parabolic barrier very well approxi- 
mates those potential shapes with $\lambda \cong 0.07$, almost identical curves, with similar values for $T_{1}$ and $T_{0}$, were obtained. In the same reference it has also been shown that the field and the temperature dependences of $j_{H}$ are in good agreement with the theoretical predictions. Furthermore, the values of $T_{1}$ and $T_{0}$ derived from the high-field behaviors are consistent with those generated by fitting the low-field $\rho(T)$ data, indicating that the characteristics of $\rho(T)$ and $j_{H}$ indeed originate from a common mechanism. It may be asked whether some other effects, such as FrenkelPoole or Fowler-Nordheim tunneling, can generate the same observed characteristics. To answer this question, we note that the high- and the low-field behaviors of the C-PVC conductivity are easily distinguished from the $\ln \rho^{\sim} \mathcal{E}^{1 / 2} / T$ dependence of the Frenkel-Poole effect and the $\ln j_{H} \sim 1$ / $\mathcal{E}$ dependence for the Fowler-Nordheim tunneling. Hopping mechanisms ${ }^{1,2}$ can also be excluded, because no law of the form $\exp \left(b / T^{\alpha}\right)$ (for a single value of $\alpha$ ) describes the resistivity of all the carbon samples. Hopping conduction in granular metals further differs from the fluctuation-induced tunneling in that the two conduction processes yield different field dependences for $j_{H}$.

\section{B. Disordered semiconductors}

It is generally agreed that the nature of electronic states in the energy band tails plays a dominant role in the electrical properties of disordered semiconductors. In this section we focus our attention on a particular class of disordered semiconductor, heavily doped, closely compensated, crystalline semiconductor, ${ }^{4}$ in which the energy band tails are the result of random potential fluctuations associated with heavy doping. To describe the conduction process in these materials, it is helpful to picture the random potential fluctuations as the surface contour of a rugged lake bed. ${ }^{4}$ In this analog the Fermi level corresponds directly to the water level of the lake. Depending on the position of the Fermi (water) level, which can be controlled by adjusting the level of compensation, three conduction regimes are distinguished. When the Fermi (water) level is low (in the energy band tail), electrons occupy isolated, localized regions (analogous to water puddles in an almost dry lake bed), and the conduction is by hopping from one localized site to another. As the Fermi (water) level is raised in energy, localized regions merge together to form continuous conducting pathways which are broken by only a few potential barriers. Electrical conduction in this case is expected to be dominated by fluctuationinduced tunneling. When the Fermi (water) level is raised further, the continuous conducting paths eventually span the whole material, and metallic conduction ensues.

For the case of heavily doped, closely compensated GaAs, various data show the conduction to be among localized states. ${ }^{4}$ However, the observed strong field dependence of the conductivity suggests that the localized regions are large in size so that the field is magnified by confining all the applied potential to the small regions separating the conducting pathways. Therefore, out of the three conduction mechanisms described above, fluctuation-induced tunneling seems to be the most appropriate for the present material. ${ }^{30}$

Figure 9 shows $\rho$ vs $1 / T$ for a sample ${ }^{4}$ of disordered GaAs. As pointed out by Redfield, ${ }^{31}$ the data do not follow the $\ln \rho \sim 1 / T^{1 / 4}$ dependence of the variable range hopping. However, by using Eq. (28) with $\lambda \simeq 0.02, T_{1}=280 \mathrm{~K}, T_{0}=16.5 \mathrm{~K}$, good agreement is obtained. From the values of $\lambda, T_{1}$, and $T_{0}$, one can calculate the median values of the tunnel junction parameters. By taking $V_{0} \cong 0.05$ $\mathrm{eV}$ estimated from density -of-states considerations $s^{4}$ and using the free electron mass in the calculation of $\chi$, we get $w \simeq 70 \AA$ and $A \cong 1700 \AA^{2}$.

The high-field behavior of $j_{H}$ for the same sample is shown in Fig. 10. The solid lines are calculated using Eq. (30a) with the values of $\lambda, T_{1}$, and $T_{0}$ obtained from the temperature dependence of resistivity. We have also assumed that $\epsilon=1$ (i.e., $\mathcal{E}=\mathcal{E}_{0}$ ) occurs at $\mathcal{E}=250 \mathrm{~V} / \mathrm{cm}$ and that $j_{00}$

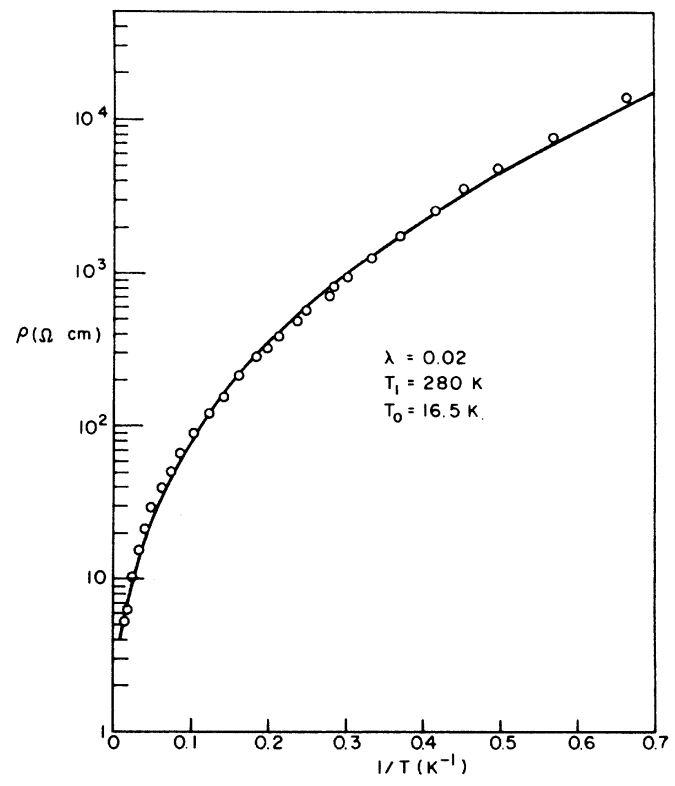

FIG. 9. Resistivity of heavily doped, closely compensated GaAs as a function of $1 / T$. The solid line is calculated from Eq. (28) with the parameter values given in the figure. 


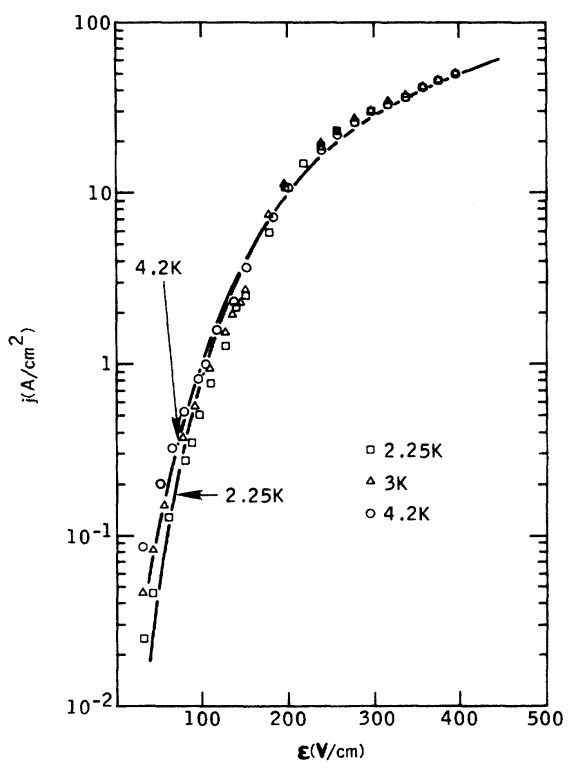

FIG. 10. The field dependence of the current density for the same sample of GaAs whose resistivity data are shown in Fig. 9. The solid lines are calculated from Eq. (30a) with the same parameter values as those given in Fig. 9. The curve for $T=3 \mathrm{~K}$, which lies between those for 2.25 and $4.2 \mathrm{~K}$, is not plotted in order to avoid cluttering.

$=0.14 \mathrm{~A} / \mathrm{cm}^{2}$. The relatively small field strength at which the non-ohmic behavior appears is an indication of the long length of the conducting pathways as stated earlier. It is seen that the experimental values of the current density at different temperatures tend to merge together at high fields as predicted by the theory, and the general agreement between theory and experiment is excellent, especially in view of the fact that the parameters $\lambda, T_{1}$, and $T_{0}$ are all fixed by the low-field temperature dependence of the resistivity.

\section{Doped polyacetylene}

Polyacetylene, $(\mathrm{CH})_{x}$, is an organic semiconductor which exists in the form of linear polymeric chains. When doped with controlled amounts of halogens, such as chlorine, bromine, iodine, or with $\mathrm{AsF}_{5}$, films of this semiconducting polymer show large increase in the electrical conductivity. At the dopant concentration of $\sim 1$ at. $\%$, a semiconductor-metal transition occurs. ${ }^{5,6}$ Far-infrared measurements and thermoelectric power studies reveal that above this critical dopant concentration the $(\mathrm{CH})_{x}$ exhibits metallic behaviors such as free carrier absorption and positive, linear Seebeck coefficient as a function of temperature. However, the resistivity of the doped $(\mathrm{CH})_{x}$ in the metallic regime has been observed to decrease as $T$ increases, which is distinctly a nonmetallic

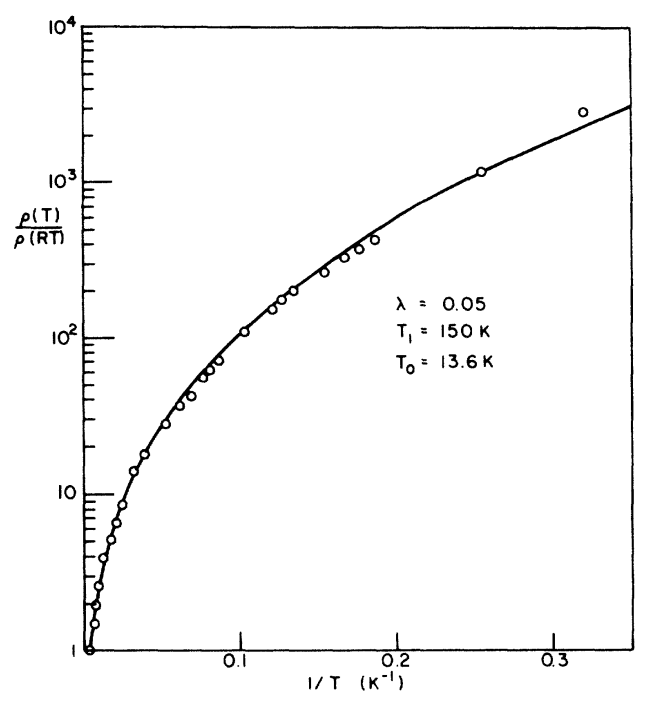

FIG. 11. Normalized resistivity of doped polyacetylene in the metallic regime plotted as a function of $1 / T$. $\rho(\mathrm{RT})$ denotes the value of resistivity at room temperature. The solid line is calculated from Eq. (28) with the parameter values given in the figure.

characteristic. In order to understand this apparent contradiction, it is essential to take into consideration that the $(\mathrm{CH})_{x}$ films consist of tangled, randomly oriented fibrils. Therefore, whereas the fibrils themselves are metallic, the electrical conductivity may be dominated by electron tunneling between the conducting segments. It follows that the theory of fluctuation-induced tunneling should be descriptive of the temperature-dependence of resistivity in doped $(\mathrm{CH})_{x}$.

In Fig. 11 the normalized resistivity data of Chiang et al. ${ }^{5}$ for $\left(\mathrm{CHI}_{0,22}\right)_{x}$ are plotted as a function of $1 / T$. The solid line is the theoretical fit using Eq. (28) with $^{32} \lambda=0.05, T_{1}=150 \mathrm{~K}$, and $T_{0}$ $=13.6 \mathrm{~K}$. The good agreement between the theory and the experimental results demonstrates that the nonmetallic behavior of the resistivity can indeed be reconciled with the intrinsic metallic nature of the doped $(\mathrm{CH})_{x}$ polymer chains. Moreover, since the intrinsic metallic resistivity of the $(\mathrm{CH})_{x}$ should increase as a function of temperature, the present model predicts a resistivity minimum near the temperature where the tunneling resistance equals the intrinsic metallic resistance (treating the two as in series). It is suggested that this could be the explanation for the $(\mathrm{CH})_{x}$ resistivity minimum observed by Seeger et al. ${ }^{6}$

\section{SUMMARY}

By considering the effects induced by thermally activated voltage fluctuations as an integral part 
of the tunneling process, it is shown in this paper that the fluctuation-induced tunneling conductivity is thermally activated at high temperatures but becomes identical to simple (temperature-independent) elastic tunneling at low temperatures. Between the two limiting behaviors the temperature dependence of the conductivity is controlled by the shape of the tunneling barrier. The tunneling current is also found to be a nonlinear increasing function of the electric field. However, the degree of nonlinearity decreases as the temperature increases, which indicates that the tunnel barrier is effectively lowered and narrowed by the fluctuating voltages. The generalization of the theory from the consideration of a single tunnel junction to a random network of tunnel junctions is achieved through the application of the effective-medium theory. For peaked distributions of the junction parameters $T_{1}$ and $T_{0}$ it is demonstrated that the resulting network conductivity can be well represented by single-junction characteristics. The theory is applied to three distinct disordered systems where the conductivity is believed to be dominated by electron tunneling across small gaps separating long (large) conducting pathways (regions). In each case excellent agreement is obtained with the experimental results.

\section{ACKNOWLEDGMENTS}

The author wishes to thank E. K. Sichel, J. I. Gittleman, R. W. Cohen, and C. H. Anderson for many useful discussions in the course of this work, J. Matey for help in digitizing the data shown in Fig. 11, and D. A. de Wolf for pointing out that the function $\varphi(\epsilon)$ can be accurately represented by the rational approximation Eq. (32a).

\section{APPENDIX A}

In this appendix we investigate the effect of voltage fluctuations on the parallel conduction mechanism of thermal activation over the potential barrier. For the image-force corrected barrier, $V$ $=V_{0}\left[1-\lambda / u(1-u)-\overline{\mathcal{E}}_{0} \epsilon u\right]$, the maximum height of the barrier $V_{m}$ is a function of the electric field,

$$
V_{m}(\epsilon)=V_{0}(1-4 \lambda) h(\epsilon),
$$

where the function $h(\epsilon)$ is shown in Fig. 12 for several values of $\lambda$. Since for a given value of $\epsilon$ the probability $Q$ of thermal activation over the barrier is given by

$$
Q= \begin{cases}\exp \left(-V_{m} / k T\right) & \text { if } \epsilon \leqslant 1 \\ 1 & \text { if } \epsilon>1,\end{cases}
$$

the conductivity by thermal activation can be expressed as

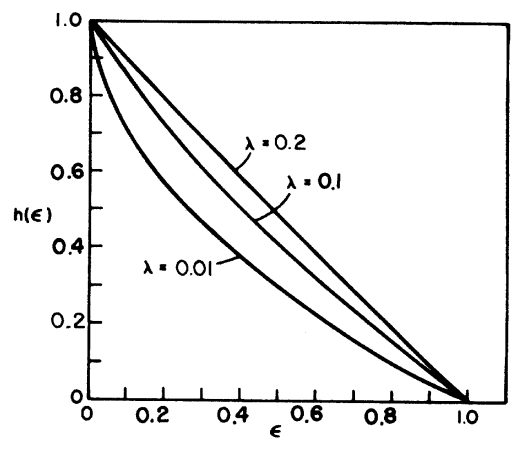

FIG. 12. The function $h(\epsilon)$, which describes the variation of the normalized barrier height, plotted for three different values of $\lambda$.

$$
\sigma_{\text {th. act. }} \propto \int_{0}^{\infty} d \epsilon Q(\epsilon) P(\epsilon),
$$

where $P(\epsilon)=\left(4 T_{1} / \pi T\right)^{1 / 2} \exp \left(-T_{1} \epsilon^{2} / T\right)$ is the probability of occurrence of $\epsilon$ by voltage fluctuations. By writing $k T_{v}=V_{0}(1-4 \lambda)$, Eq. (A3) becomes

$$
\sigma_{\text {th. act. }} \propto\left(\frac{4 T_{1}}{\pi T}\right)^{1 / 2} \int_{0}^{\infty} d \epsilon \exp \left(-\frac{T_{v} H(\epsilon)+T_{1} \epsilon^{2}}{T}\right),
$$

where

$$
H(\epsilon)= \begin{cases}h(\epsilon), & \epsilon \leqslant 1 \\ 0, & \epsilon>1\end{cases}
$$

The integrand of Eq. (A4a) is sharply peaked at $\epsilon=\epsilon_{m}$, where $\epsilon_{m}$ denotes the location of the maximum value for $-\left[T_{v} H(\epsilon)+T_{1} \epsilon^{2}\right]$. As a result the dominant temperature dependence of $\sigma_{\text {th. act. }}$ can be obtained by expanding the exponent around its maximum and performing the integration

$$
\sigma_{\text {th. act. }} \propto \exp \left(\frac{T_{v} H\left(\epsilon_{m}\right)+T_{1} \epsilon_{m}^{2}}{T}\right) .
$$

The slope of $h(\epsilon)$ is a monotonically increasing function of $\epsilon$. Therefore, if $2 T_{1} \leqslant\left|T_{v} h^{\prime}(1)\right|$, the maximum value of $-\left[T_{v} H(\epsilon)+T_{1} \epsilon^{2}\right]$ would always occur at $\epsilon_{m}=1$, which implies

$$
\sigma_{\text {th. act. }} \propto \exp \left(-T_{1} / T\right) \text {. }
$$

Since for the materials considered in this paper we have $\left|T_{v} h^{\prime}(1)\right| Z 2 T_{1}$, it is expected that conduction by thermal activation should have the same temperature dependence, $\exp \left(-T_{1} / T\right)$, as fluctuation-induced tunneling at high temperatures but becomes negligible at low temperatures as stated in Sec. III.

In order to get some idea about the temperature and the field dependences of $\sigma_{\mathrm{th} \text {. act. }}$ for arbitrary values of $T_{1}$ and $T_{v}$, it would be convenient to use 
the parabolic barrier approximation [see Eq. (33)]. In that case $V_{m}$ is given by

$$
V_{m}(\epsilon)=\left(V_{0} / 4\right)(1-\epsilon)^{2} \text {. }
$$

Defining $k T_{v}=V_{0} / 4$, we can rewrite Eq. (44a) as

$\sigma_{\text {th. act. }}$

$$
\propto\left(\frac{4 T_{1}}{\pi T}\right)^{1 / 2} \int_{0}^{\infty} d \epsilon \exp \left[-\frac{T_{v}\left[1-\left(\epsilon+\epsilon_{A}\right)\right]^{2}+T_{1} \epsilon^{2}}{T}\right],
$$

where the applied field $\epsilon_{A}$ is included as a part in the total field. By expanding the exponent and performing the integral, we get

$$
\sigma_{\text {th. act. }} \propto \exp \left[-\frac{1}{T} \frac{T_{v} T_{1}}{T_{v}+T_{1}}\left(1-\epsilon_{A}\right)^{2}\right] .
$$

Comparing this expression with Eq. (36b) shows that there is a temperature $T_{c}=T_{0} T_{v} / T_{1}$ above which $\sigma_{\text {th. act. }}$ dominates over fluctuation-induced tunneling conductivity (treating the two conduction mechanisms as parallel). However, if $T_{v} \gg T_{1}$, then $T_{c} \gg T_{0}, T_{v} T_{1} /\left(T_{v}+T_{1}\right) \cong T_{1}$, which means $\sigma_{\text {th. act. }}$ is indistinguishable from the fluctuationinduced tunneling conductivity above $T_{c}$. Therefore, in this case the consideration of fluctuationinduced tunneling alone is sufficient to account for the conduction characteristics of the junction at all temperatures. For $T_{v} \lessgtr T_{1}$, the activation energy of $\sigma_{\text {th. act. }}, k T_{v} T_{1} /\left(T_{v}+T_{v}\right)$, is smaller than both $k T_{1}$ and $k T_{v}$. This is the regime where the size of the junction is just small enough to effect a significant reduction in barrier height by voltage fluctuations. When the junction is macroscopic in size, $T_{1} \gg T_{v}, T_{v} T_{1} /\left(T_{v}+T_{1}\right) \cong T_{v}$, and

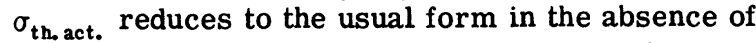
voltage fluctuations. We should also note that since $T_{1} / T_{0}=B \chi w$ [see Eq. (27c)], $B$ being some numerical constant, $T_{c}=T_{v} / B \chi w$ in this case is exactly the temperature above which the thermally activated conduction [given by $\exp \left(-T_{v} / T\right)$ ] dominates over simple tunneling [given by $\exp (-B \times w)$ ] for a macroscopic junction.

\section{APPENDIX B}

This appendix examines the probability of thermally activated tunneling for electrons with energy $E\left(<V_{m}\right)$ above the Fermi level. By combining the occupation probability of the electron, which is essentially $\exp (-E / k T)$ for $E>0$, and the barrier transmission factor $\exp [-F(E)]$, where $F(E)$ is given by Eq. (12) with $\epsilon=0$, we obtain the probability of thermally activated tunneling as

$$
\begin{aligned}
& P(E)=\exp [G(E)], \\
& G(E)=-E / k T-F(E) .
\end{aligned}
$$

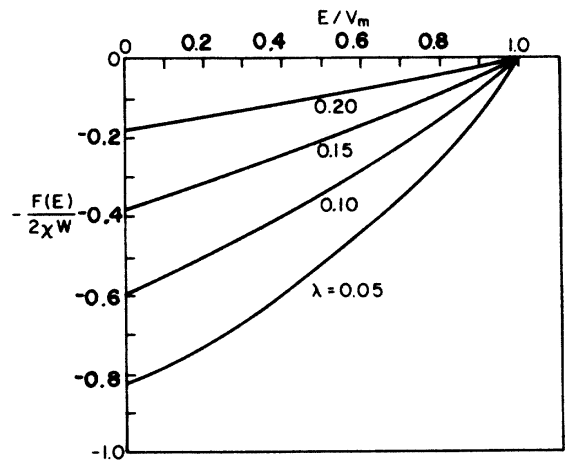

FIG. 13. The function $-F(E) / 2 \chi w$ plotted as a function of $E / V_{m}$ for five different values of $\lambda$.

For the image-force corrected barrier, $-F(E)$ is plotted in Fig. 13 for several values of $\lambda$. It is noted that the slope of $-F(E)$ is positive and is a monotonically increasing function of $E$ (this fact is not changed when $\epsilon \neq 0$ ). Therefore, if $1 / k T$ is smaller than $-F^{\prime}(0)$, then $G^{\prime}(E)>0$ for $0<E<V_{m}$ and the maximum value of $G(E)$ occurs at $E=V_{m}$, implying that thermal activation over the barrier is the dominant conduction process. When $1 / k T$ $>F^{\prime}(0)$, the function $G(E)$ develops a minimum at $0<E<V_{m}$. However, the maximum value of $G(E)$ occurs either at $E=0$ or $E=V_{m}$, which means that, depending on the temperature, either tunneling at the Fermi level or thermal activation over the barrier may be dominant, but in no temperature range does the thermally activated tunneling ever become dominant. These considerations lead directly to the conclusion that as long as tunneling is the dominant mechanism, it is permissible to treat the Fermi level as being sharp (by neglecting the contributions of those electrons in the exponential tail above $E=0$ ), since the probability of thermally activated tunneling decreases exponentially away from $E=0$.

\section{APPENDIX C}

The validity of treating $\sigma_{0}$ as a constant and the accuracy of the approximation expressed by Eq. (31a) are investigated in this appendix.

To evaluate $\sigma_{0}$ from Eq. (29), it is first necessary to calculate the quantities $\Sigma_{0}(\sigma)$ and $\Sigma_{1}(\epsilon)$ from their definitions, Eqs. (26b) and (26c). This can be done numerically by programming the expressions for $\xi(\epsilon)$ [Eq. (17b)], $\eta_{0}(\epsilon)$ [Eq. (17c)], and $\eta_{1}(\epsilon)$ [Eq. (21b)] on computer. As an explicit illustration, the case for $\lambda=0.1, \chi w=10$ is calculated. In this case $\overline{\mathcal{E}}_{0}=1.89659$ (see Fig. 4), $\Sigma_{0}(\epsilon) / \Sigma_{00}$ decreases monotonically from 11.5 at $\epsilon=0$ to 9.4 at $\epsilon=1$, and $\Sigma_{1}(\epsilon) / \Sigma_{00} \cong 10.76+45.64$ $(\epsilon-1)$. By using the calculated values of $\Sigma_{0}(\epsilon)$, $\Sigma_{1}(\epsilon)$, and $T_{1}=12.096 T_{0}[\xi(0)=0.6408$ for $\lambda=0.1]$, 


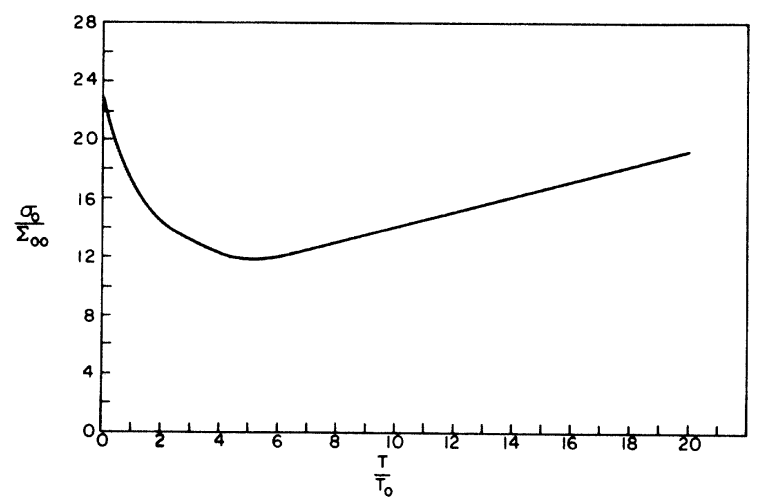

FIG. 14. The temperature dependence of $\sigma_{0} / \Sigma_{00}$ for $\lambda=0.1$.

the integrals of Eq. (27a) can be numerically evaluated as a function of $T / T_{0}$. The results are plotted in Fig. 14. It is seen that compared to the exponential term, $\sigma_{0}$ is indeed a slowly varying function of temperature as expected. Since similar results hold for other values of $\lambda$ as well, it can be concluded that treating $\sigma_{0}$ as a constant in Eq. (28) is an excellent approximation.

The expressions for $j_{H}$, Eq. (30a), can be numerically calculated in the same fashion as above. For $\lambda=0.1$, the comparison between Eq. (30a) and its approximation, Eq. (31), is shown in Fig. 15. At $T / T_{0} \leqslant 2$, the $j_{H}$ field dependences of Eqs. (30a) and (31) are similar for $\epsilon_{A} \leqslant 0.5$, indicating that in this regime the field dependence is dominated by the variation in $\epsilon^{+}$. However, when $\epsilon_{A} \geqslant 0.5$, or $T / T_{0} \geqslant 2$, the second integral in Eq. (30a) becomes important, and significant deviations from the actual value of $j_{H}$ are observed. Instead of merging together at $\epsilon_{A}=1$ and $j_{H}=j_{H}^{0}$ as approximated by Eq. (31), curves of $j_{H}(\epsilon)$ at different temperatures tend to come together at some point with $\epsilon_{A} \simeq 1.5$ and $j_{H} \geqslant j_{H^{*}}^{0}$. This comparison demonstrates that while Eq. (31) catches the essential qualitative features of $j_{H}$, quantitatively the errors resulting from the approximation could be nonnegligible. Therefore, it is expected that the use of Eq. (31) to fit the $j_{H}$ data would yield slightly distorted values for the parameters $\lambda, T_{1}$, and $T_{0}$.

\section{APPENDIX D}

In this appendix we formulate an approximation for the function $\varphi(\epsilon)$. Since the function $\varphi(\epsilon)$ is defined for $0 \leqslant \epsilon \leqslant 1$ with $\varphi(0)=1, \varphi(1)=0$, a

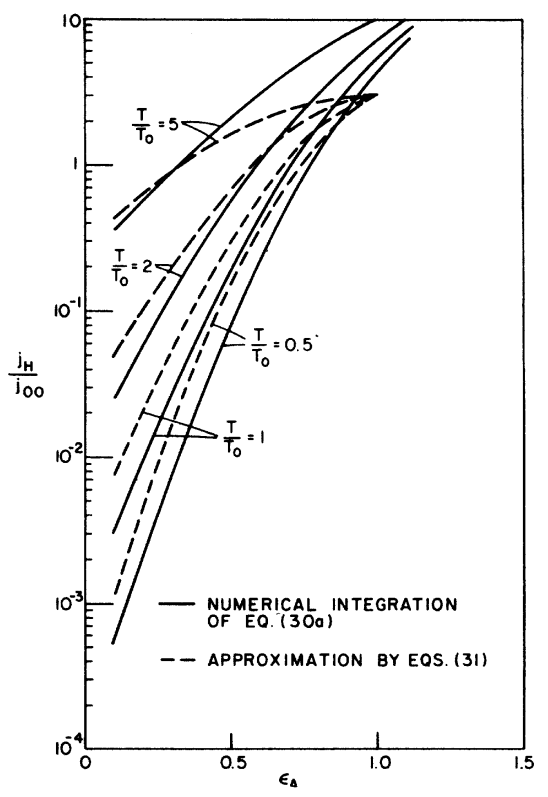

FIG. 15. Comparison of Eq. (30a) with its approximation, Eqs. (31).

rational approximation in the form

$$
\varphi(\epsilon) \cong(1-\epsilon) /\left(1+\alpha \epsilon+\beta \epsilon^{2}\right)
$$

is seen to satisfy the boundary requirements. To determine $\alpha$ and $\beta$, we note that

$$
\begin{aligned}
& \varphi^{\prime}(0)=-(1+\alpha), \\
& \varphi^{\prime}(1)=-(1+\alpha+\beta)^{-1} .
\end{aligned}
$$

Equations (D2) can be solved for $\alpha$ and $\beta$, yielding

$$
\begin{aligned}
& \alpha=-\left[\varphi^{\prime}(0)+1\right], \\
& \beta=-\left(\frac{1}{\varphi^{\prime}(1)}+\varphi^{\prime}(0)\right) .
\end{aligned}
$$

Values of $\varphi^{\prime}(0)$ and $\varphi^{\prime}(1)$ are numerically evaluated as a function of $\lambda$. It is found that

$$
\begin{aligned}
& \alpha=4.2837 \times 10^{-4}\left(\frac{1}{\lambda}-4\right)^{2}+4.8728 \times 10^{-2}\left(\frac{1}{\lambda}-4\right), \\
& \beta=\operatorname{coth}\left(\frac{2.6 \lambda}{1-4 \lambda}\right)-(1+\alpha),
\end{aligned}
$$

give excellent account of the variation of $\alpha$ and $\beta$ with respect to $\lambda$. Therefore, by using the above formulas for $\alpha$ and $\beta$ in conjunction with Eq. (B1), the function $\varphi(\epsilon)$ can be evaluated accurately without resorting to numerical integration.

\footnotetext{
* Present address: Theoretical Sciences Group, Corporate Research Center, Exxon Research \& Engineering Co., P. O. Box 45, Linden, NJ 07036.
}

${ }^{1}$ N. F. Mott, Philos. Mag. 19, 835 (1969); V. Ambegaokar, B. I. Halperin, and J. S. Langer, Phys. Rev. B $\underline{4}$, 2612 (1971). 
${ }^{2}$ P. Sheng, B. Abeles, and Y. Arie, Phys. Rev. Lett. 31, 44 (1973); B. Abeles, P. Sheng, Y. Arie, and M. Coutts, Adv. Phys. 24, 407 (1975); P. Sheng and B. Abeles, Phys. Rev. Lett. 28, 34 (1972).

${ }^{3}$ P. Sheng, E. K. Sichel, and J. I. Gittleman, Phys. Rev. Lett. 40, 1197 (1978); E. K. Sichel, J. I. Gittleman, and P. Sheng, Phys. Rev. B 18, 5712 (1978).

${ }^{4}$ D. Redfield, Adv. Phys. 24, 463 (1975).

${ }^{5}$ C. K. Chiang, C. R. Fincher, Jr., Y. W. Park, A. J. Heeger, H. Shirakawa, E. J. Louis, S. C. Gau, and A. G. MacDiarmid, Phys. Rev. Lett. 39, 1098 (1977); C. K. Chiang, Y. W. Park, A. J. Heeger, H. Shirakawa, E. J. Louis, and A. G. MacDiarmid, J. Chem. Phys. (to be published).

${ }^{6}$ K. Seeger, W. D. Gill, T. C. Clarke, and G. B. Street, Solid State Commun. 28, 873 (1978); R. L. Greene, T. C. Clarke, W. D. Gill, P. M. Grant, J. F. Kwak, and G. B. Street, report presented at NATO ASI, Les Arcs, France, 1978 .

${ }^{7}$ This is the classical expression for the Johnson noise. To get the quantum-mechanical expression, $k T$ has to be replaced by $I(f)=h f\left\{\frac{1}{2}+1 /[\exp (h f / k T)-1]\right\}$ [see H. B. Callen and T. A. Welton, Phys. Rev. 83,34 (1951); W. Bernard and H. B. Callen, Rev. Mod. Phys. $31,1017(1959)$ ]. When $I(f)$ is substituted in place of $\overline{k T}$ in Eq. (1a), however, a divergence is obtained due to the existence of the $h f / 2$ term in $I(f)$. According to A. van der Ziel (private communications), this divergence can be avoided if the resistor $R$ has a small amount of inductance. The total fluctuating voltage then saturates at a finite value. An alternative form for $I(f)$, i.e., without the $h f / 2$ term, has also been proposed [see H. Nyquist, Phys. Rev. 29, 614 (1927)]. In that case $\left\langle V_{T}{ }^{2}\right\rangle$ calculated from Eq. $(1 \mathrm{a})$ is a function of $R$, and there is a character istic temperature $T=\hbar / R C k$ below which the value of $\left\langle V^{2}\right\rangle$ would fall short of $k T / C$ as given by Eq. (1b). If such effects do exist, their manifestation in the temperature dependence of the conductivity would be difficult to distinguish from those caused by a slight change in the barrier shape (see Fig. 6). In the literature, some doubts about the validity of the quantum corrections was also raised [D. K. C. MacDonald, Physica (Utrecht) 28, 409 (1962)]. At present no experimental verification for either form of the quantum correction has been achieved. The author wishes to acknowledge useful discussions of the quantum correction question with D. Southgate.

${ }^{8}$ See, for example, D. K. C. MacDonald, Noise and Fluctuations: an Introduction (Wiley, New York, 1962).
${ }^{9}$ See, for example, R. P. Feynman, R. B. Leighton, and M. L. Sands, Feynman Lectures on Physics, (AddisonWesley, Reading, Mass., 1963), Vol. I, Chap. 41.

${ }^{10} \mathrm{~A}$ more rigorous derivation can be found in N. G. van Kampen, Physica (Utrecht) 26, 585 (1960).

${ }^{11}$ W. Schottky, Z. Phys. 14, 63 (1923).

${ }^{12} \mathrm{~A}$. Sommerfeld and $\mathrm{H}$. Bethe, Handb̈̈̈ch der Physik, edited by H. Geiger and K. Scheel (Springer, Berlin, 1933).

${ }^{13}$ R. H. Fowler and L. W. Nordheim, Proc. R. Soc. London, Sect. A 119, 173 (1928).

${ }^{14} \mathrm{~W}$. R. Smythe, Static and Dynamic Electricity (McGraw-Hill, New York, 1950), Chap. IV.

${ }^{15}$ J. G. Simmons, J. Appl. Phys. 34, 1793 (1963).

${ }^{16}$ See, for example, C. B. Duke, Tunneling in Solids (Academic, New York, 1969), Chap. IV.

${ }^{17}$ E. C. Kemble, Fundamental Principles of Quantum Mechanics (McGraw-Hill, New York, 1937), Chap. III.

${ }^{18}$ L. D. Landau and E. M. Lifshitz, Quantum MechanicsNon-Relativistic Theory (Addison-Wesley, Reading, Mass. 1958), Sec. 50.

${ }^{19}$ S. C. Miller, Jr. and R. H. Good, Jr., Phys. Rev. 91, 174 (1953).

${ }^{20}$ E. L. Murphy and R. H. Good, Jr., Phys. Rev. 102, 1464 (1956).

${ }^{21}$ R. H. Good, Jr. and E. W. Müller, in Handbüch der Physik, edited by S. Flugge (Springer, Berlin, 1956), Vol. 21 , p. 176.

${ }^{22}$ R. Holm and B. Kirschstein, Z. Tech. Phys. 16, 488 (1935).

${ }^{23}$ S. Kirkpatrick, Rev. Mod. Phys. 45, 574 (1973). ${ }^{24} \mathrm{~J}$. Bernasconi, Phys. Rev. B 7, 2252 (1973).

${ }^{25}$ C. G. Granqvist and R. A. Buhrman, J. Appl. Phys. 47, 2200 (1976).

${ }^{26}$ L. P. Fox, RCA Rev. 39, 116 (1978).

${ }^{27}$ For information regarding the manufacturing companies of these carbons, see Ref. 3 .

${ }^{28} \mathrm{M}$. D. Coutts (unpublished).

${ }^{29}$ W. W. Tyler and A. C. Wilson, Jr., Phys. Rev. 89, 870 (1953).

${ }^{30}$ To be more accurate, the fluctuation-induced tunneling should only be applicable at low temperatures. As discussed in Ref. 4 , at $T \geqslant 50 \mathrm{~K}$ the electrons occupying high-mobility states above the Fermi level contribute a non-negligible amount to the conductivity.

${ }^{31} \mathrm{D}$. Redfield, Phys. Rev. Lett. 30, 1319 (1973).

${ }^{32}$ The knowledge of $\lambda, T_{1}$, and $T_{0}$ is not sufficient to uniquely determine the mean values of the barrier parameters $V_{0}, w$, and $A$. However, if we assume $V_{0}=0.1 \mathrm{eV}$, then $w=38 \AA$ and $A=890 \AA^{2}$. 